\title{
A photometric and spectroscopic study of the new dwarf spheroidal galaxy in Hercules $\star, \star \star$
}

\section{Metallicity, velocities, and a clean list of RGB members}

\author{
D. Adén ${ }^{1}$, S. Feltzing ${ }^{1}$, A. Koch ${ }^{2}$, M. I. Wilkinson ${ }^{2}$, E. K. Grebel ${ }^{3}$, I. Lundström ${ }^{1}$, G. F. Gilmore ${ }^{4}$, D. B. Zucker ${ }^{5,6}$, \\ V. Belokurov ${ }^{4}$, N. W. Evans ${ }^{4}$, and D. Faria ${ }^{4}$ \\ 1 Lund Observatory, Box 43, 22100 Lund, Sweden \\ e-mail: daniel.aden@astro.lu.se \\ 2 Department of Physics and Astronomy, University of Leicester, University Road, Leicester LE1 7RH, UK \\ 3 Astronomisches Rechen-Institut, Zentrum für Astronomie der Universität Heidelberg, Mönchhofstr. 12-14, 69120 Heidelberg, \\ Germany \\ 4 Institute of Astronomy, Madingley Road, Cambridge, CB3 0HA, UK \\ 5 Department of Physics, Macquarie University, North Ryde, NSW 2109, Australia \\ 6 Anglo-Australian Observatory, PO Box 296, Epping, NSW 1710, Australia
}

Received 18 June 2009 / Accepted 13 August 2009

\section{ABSTRACT}

\begin{abstract}
Context. Dwarf spheroidal (dSph) galaxies are the least luminous, least massive galaxies known. Recently, the number of observed galaxies in this class has greatly increased thanks to large surveys. Determining their properties, such as mass, luminosity and metallicity, provides key information in our understanding of galaxy formation and evolution.

Aims. Our aim is to provide as clean and as complete a sample as possible of red giant branch stars that are members of the Hercules dSph galaxy. With this sample we explore the velocity dispersion and the metallicity of the system.

Methods. Strömgren photometry and multi-fibre spectroscopy are combined to provide information about the evolutionary state of the stars (via the Strömgren $c_{1}$ index) and their radial velocities. Based on this information we have selected a clean sample of red giant branch stars, and show that foreground contamination by Milky Way dwarf stars can greatly distort the results.

Results. Our final sample consists of 28 red giant branch stars in the Hercules dSph galaxy. Based on these stars we find a mean photometric metallicity of $-2.35 \pm 0.31$ dex which is consistent with previous studies. We find evidence for an abundance spread. Using those stars for which we have determined radial velocities we find a systemic velocity of $45.20 \pm 1.09 \mathrm{~km} \mathrm{~s}^{-1}$ with a dispersion of $3.72 \mathrm{~km} \mathrm{~s}^{-1}$, this is lower than values found in the literature. Furthermore we identify the horizontal branch and estimate the mean magnitude of the horizontal branch of the Hercules dSph galaxy to be $V_{0}=21.17 \pm 0.05$, which corresponds to a distance of $147_{-7}^{+8} \mathrm{kpc}$

Conclusions. When studying sparsely populated and/or heavily foreground contaminated $\mathrm{dSph}$ galaxies it is necessary to include knowledge about the evolutionary stage of the stars. This can be done in several ways. Here we have explored the power of the $c_{1}$ index in Strömgren photometry. This index is able to clearly identify red giant branch stars redder than the horizontal branch, enabling a separation of red giant branch dSph stars and foreground dwarf stars. Additionally, this index is also capable of correctly identifying both red and blue horizontal branch stars. We have shown that a proper cleaning of the sample results in a smaller value for the velocity dispersion of the system. This has implications for galaxy properties derived from such velocity dispersions.
\end{abstract}

Key words. galaxies: dwarf - galaxies: fundamental parameters - galaxies: individual: Hercules galaxies: kinematics and dynamics - galaxies: photometry

\section{Introduction}

In the past few years the number of known Milky Way satellites has increased considerably. Our Galaxy has gained at least ten newly recognized companions, and additional ones await confirmation (e.g. Zucker et al. 2006a,b; Walsh et al. 2007; Belokurov et al. 2006, 2007, 2008, 2009). These recently discovered satellites resemble the previously known dwarf spheroidal (dSph)

\footnotetext{
* Based on observations made with the INT telescope operated on the island of La Palma by the Isaac Newton Group in the Spanish Observatorio del Roque de los Muchachos of the Instituto de Astrofisica de Canarias.

$\star \star$ Based on spectra obtained with the VLT-U2 telescope ESO Proposal number 079.B-0447(A).
}

galaxies in many of their characteristics (see Grebel et al. 2003, for a detailed discussion of the properties of the classical dSph galaxies), but most of the new satellites are several magnitudes fainter than any dwarf galaxy known before. Hence these objects are now often referred to as "ultra-faint dwarf spheroidal galaxies". Since most of the new discoveries were made using deep CCD sky surveys such as the Sloan Digital Sky Survey, which cover primarily the northern hemisphere, it seems highly likely that additional objects will be added once the southern sky is scanned in a similar manner (e.g. Keller et al. 2007; Walsh et al. 2009).

One of the new discoveries is the Hercules dwarf spheroidal galaxy (Belokurov et al. 2007). Hercules lies at a distance of $132 \pm 12 \mathrm{kpc}$ from us, has an absolute $V$-band magnitude of 
about $-6.6 \pm 0.3$, a $V$-band surface brightness of only $27.2 \pm$ $0.6 \mathrm{mag} \operatorname{arcsec}^{-2}$, and appears highly elongated (Coleman et al. 2007; Martin et al. 2008). Its stellar mass is estimated to be in the range of several $\times 10^{4} M_{\odot}$. The mass of the Hercules $\mathrm{dSph}$ galaxy, as inferred from line-of-sight radial velocity measurements, is of the order of $10^{7} M_{\odot}$ within the central $300 \mathrm{pc}$ (Strigari et al. 2008). This value is in good agreement with the seemingly ubiquitous, common mass scale of the other Galactic satellites (e.g. Gilmore et al. 2007; Walker et al. 2007; Strigari et al. 2008, and references therein).

As would be expected from its low luminosity and low surface brightness, Hercules is a metal-poor dSph galaxy. From measurements of the $\mathrm{Ca}$ II IR triplet lines in red giant stars, Simon \& Geha (2007) inferred a mean metallicity of $[\mathrm{Fe} / \mathrm{H}]=$ $-2.27 \pm 0.07$ on the metallicity scale of Carretta \& Gratton (1997). Using spectrum synthesis of Fe I lines, Kirby et al. (2008b) derived a mean metallicity of $-2.58 \pm 0.51$ dex. Both studies found a wide range of metallicities among the red giant stars in Hercules, confirming the trend known from brighter $\mathrm{dSph}$ galaxies that often exhibit spreads of $1 \mathrm{dex}$ and more (e.g. Shetrone et al. 2001; Koch et al. 2006, 2007a,b).

A detailed, high-resolution abundance analysis of two red giants in the Hercules dSph galaxy revealed that the enrichment in heavy elements proceeded inhomogeneously and that corecollapse supernovae were the primary contributors to the enrichment of Hercules (Koch et al. 2008b). Evidence for such chemical inhomogeneities on small scales has also been found in the more luminous dSph galaxies (Koch et al. 2008a) and in more massive dIrr galaxies (Kniazev et al. 2005).

In our current study we explore the potential of Strömgren photometry for the study of the stellar content of ultra-faint dSph galaxies, such as the one in Hercules. In contrast to the usual broadband photometry, Strömgren photometry offers several advantages. It provides us with gravity-sensitive multicolour indices useful for distinguishing different evolutionary stages including giant-dwarf discrimination. This is a very valuable option when trying to eliminate foreground dwarfs from a giant candidate sample to be used for subsequent spectroscopy, particularly when dealing with sparse, extended, ultra-faint dSph galaxies that tend to suffer from substantial Galactic foreground contamination. Moreover, Strömgren indices offer the possibility to estimate metallicities for red giant stars. This method has been considerably refined since the early calibration attempts by Grebel \& Richtler (1992). As compared to other photometric estimates, it has the added advantage of providing age-independent metallicity estimates (e.g. Faria et al. 2007). In terms of telescope time, intermediate-band photometry is a lot cheaper than spectroscopic surveys of faint giants.

We have obtained both Strömgren photometry as well as spectroscopic observations of the Ca II IR triplet lines for stars in the field of the Hercules dSph galaxy. These observations enable a full analysis both of evolutionary stage as well as radial velocities for these stars. It turns out that knowledge about the evolutionary stage of the stars is crucial for the construction of a clean sample of red giant branch (RGB) stars in the Hercules dSph galaxy.

The paper is organized as follows: in Sect. 2 we describe the observations and data reductions for both the photometric and the spectroscopic observations. In Sect. 3 we present the colour-magnitude diagram as well as the measured radial velocities. In Sect. 4 we show how the gravity sensitive Strömgren $c_{1}$ index can be used to disentangle the Hercules dSph galaxy members from the foreground contamination, and Sect. 5 deals with membership determination based on radial velocities. Section 6

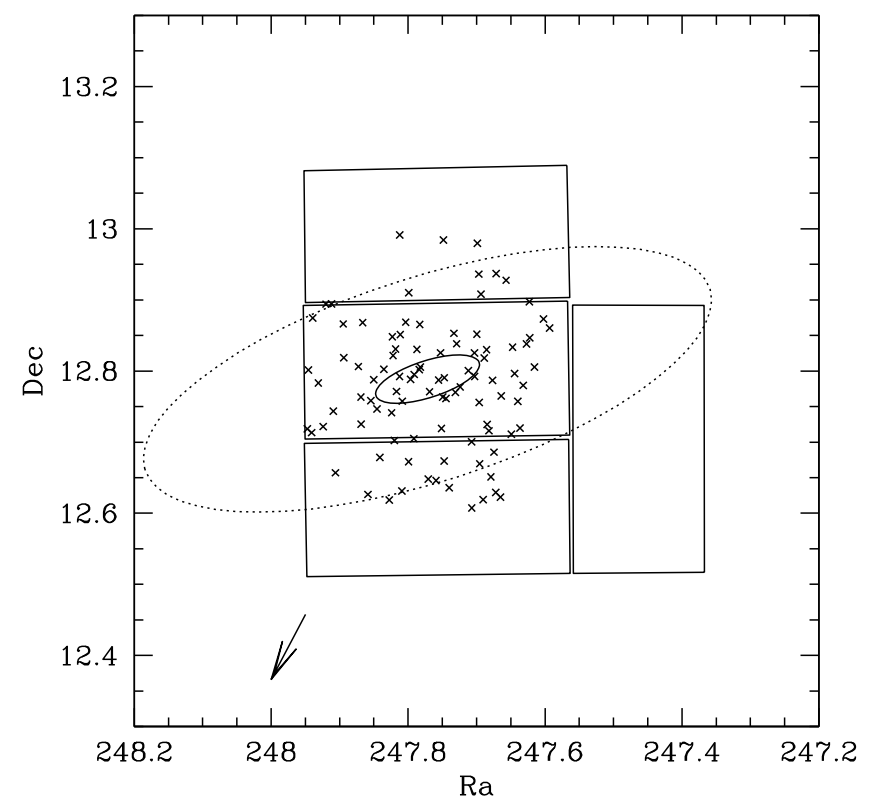

Fig. 1. Position on the sky of our observations. The coordinates are given in decimal degrees, epoch J2000. Central coordinates for the galaxy are from Martin et al. (2008). The solid ellipse represents the core radius and the dotted ellipse the King profile limiting radius of the Hercules dSph galaxy as determined by Coleman et al. (2007). Solid rectangles outline the four CCD chips in the WFC used for the photometric observations. $\times$ marks the fibre positions for the FLAMES observations. Here we show the fibres positioned on the stars only. The arrow in the bottom left hand corner indicates the direction to the centre of the Milky Way.

summarizes how we define a Hercules member star. Section 7 provides a comparison with previous velocity determinations. In Sect. 8 we derive metallicities for the stars identified as members of the Hercules dSph galaxy using the Strömgren $m_{1}$ index as well as from the measurements of the Ca II IR triplet lines, and compare the results with previous studies of the metallicity of the Hercules dSph galaxy. In Sect. 9 our results are discussed and they are summarized in Sect. 10.

\section{Observations and data reductions}

In this section we detail the observations as well as the data reduction for both photometric and spectroscopic observations.

\subsection{Photometry}

Intermediate-band Strömgren $u, v, b$ and $y$ photometry was obtained during six nights with the Wide Field Camera (WFC) on the 2.5-m Isaac Newton Telescope (INT) on La Palma. Of the six nights only three provided useful data due to bad weather conditions. The WFC consists of $42 \mathrm{k} \times 4 \mathrm{k}$ CCDs. The CCDs have a pixel size of 13.5 microns corresponding to $0.33^{\prime \prime}$ per pixel. Figure 1 shows the location and dimensions of the galaxy on the sky and the positions of the CCD chips of the WFC.

The observations are summarised in Table 1 and all observations were centered at $\mathrm{RA}=16^{\mathrm{h}} 31^{\mathrm{m}} 05^{\mathrm{s}}$ and $\mathrm{Dec}=+12^{\circ} 47^{\prime} 18^{\prime \prime}$ (Martin et al. 2008). Typical seeing during the three good nights was about 1.3 arcsec.

Multiple standard and extinction stars were observed each night. During the observations of the Hercules dSph galaxy, we observed 12 Strömgren standard stars chosen from the list in Schuster \& Nissen (1988) plus one star from Olsen (1993), see 
Table 1. Summary of the photometric observations obtained with the Isaac Newton Telescope.

\begin{tabular}{ccccc}
\hline \hline Filter & $\begin{array}{c}\text { 13 April 2007 } \\
{[\mathrm{min}]}\end{array}$ & $\begin{array}{c}\text { 14 April 2007 } \\
{[\mathrm{min}]}\end{array}$ & $\begin{array}{c}\text { 15 April 2007 } \\
{[\mathrm{min}]}\end{array}$ & $\begin{array}{c}\text { Total } \\
{[\mathrm{min}]}\end{array}$ \\
\hline$y$ & $1 \times 30$ & $1 \times 30$ & $1 \times 30$ & 90 \\
$b$ & $1 \times 30$ & $1 \times 30$ & $1 \times 30$ & 90 \\
$v$ & $1 \times 30$ & $1 \times 30$ & $1 \times 30$ & 90 \\
$u$ & $1 \times 30$ & $2 \times 30$ & $2 \times 30$ & 150 \\
\hline
\end{tabular}

Column 1 lists the filter. Columns 2-4 list the number of $30 \mathrm{~min}$ exposures obtained for each filter during each of the three useful nights. Column 5 lists the total exposure time for each filter.

Table 2. Standard stars from Schuster \& Nissen (1988), except HD107853 (Olsen 1993).

\begin{tabular}{lccccc}
\hline \hline ID & Hip & $V$ & $(b-y)$ & $m_{1}$ & $c_{1}$ \\
\hline HD 100363 & 56327 & 8.648 & 0.191 & 0.139 & 0.760 \\
HD 107853 & $\ldots$ & 9.100 & 0.321 & 0.157 & 0.472 \\
HD 108754 & 60956 & 9.006 & 0.435 & 0.217 & 0.254 \\
HD 120467 & 67487 & 8.147 & 0.728 & 0.757 & 0.088 \\
HD 134439 & 74235 & 9.058 & 0.484 & 0.224 & 0.165 \\
HD 138648 & 76203 & 8.137 & 0.504 & 0.358 & 0.290 \\
HD 149996 & 81461 & 8.495 & 0.396 & 0.164 & 0.305 \\
HD 158226 & 85378 & 8.494 & 0.386 & 0.146 & 0.316 \\
DM -05 3063 & 51127 & 9.734 & 0.568 & 0.461 & 0.182 \\
DM -08 4501 & 87062 & 10.591 & 0.452 & 0.032 & 0.274 \\
DM -12 2669 & 43099 & 10.230 & 0.229 & 0.094 & 0.490 \\
DM -13 3834 & 69232 & 10.685 & 0.415 & 0.098 & 0.183 \\
DM -14 4454 & 81294 & 10.332 & 0.565 & 0.469 & 0.192 \\
\hline
\end{tabular}

Columns 1 and 2 list the star ID and Hipparcos number, respectively. Column 3 lists the standard values adopted for the magnitude (note $y \equiv$ $V)$ and Cols. 4 to 6 the standard values adopted for the $(b-y), m_{1}$ and $c_{1}$ indices, respectively.

Table 2. Two stars from the list of standard stars were observed several times during each night in order to sample the extinction for a large range of airmass. These stars will henceforth be referred to as extinction stars.

The observations of standard and extinction stars are used to find the zeropoint, extinction coefficients and colour terms, see Sect. 2.1.2.

\subsubsection{Reduction of the photometric observations}

The images for the Hercules dSph galaxy and the standard stars were reduced with the Wide Field Survey Pipeline provided by the Cambridge Astronomical Survey Unit (Irwin \& Lewis 2001). The processing operations applied to the images were de-biasing, trimming, flatfielding, astrometry and correction for non-linearity.

\subsubsection{Standard star photometry and establishing the photometric calibration}

We obtained aperture photometry for the standard and extinction stars using the task PHOT within the IRAF ${ }^{1}$ APPHOT package. The size of the aperture was determined individually for each star by plotting the measured flux as a function of increasing

${ }^{1}$ IRAF is distributed by the National Optical Astronomy Observatories, which are operated by the Association of Universities for Research in Astronomy, Inc., under cooperative agreement with the National Science Foundation. aperture size. The aperture at which the flux no longer increased was chosen as the aperture for that star (typically 4-5 $\times F W H M$ of the stellar $p s f$ ). This curve-of-growth is used in order to maximise the signal-to-noise ratio $(S / N)$ while measuring as much flux as possible from the star (Howell 1989).

The measurements of the standard and extinction stars were used to establish the transformations needed to put our observations on the standard system of Olsen (1993). Note that the Schuster \& Nissen (1988) stars are secondary standards in this system. See Olsen (1995) for a discussion about the Olsen system as compared to the system established by Bond (1980) and Anthony-Twarog \& Twarog (1994).

The first night out of the three useful nights did not give us reliable photometry for the standard stars (i.e. there was no well defined linear relation for the transformation to the standard system for that night), and this night was thus excluded from the calibration.

First, we derived preliminary extinction coefficients, $k_{i}$, and zeropoints, $z_{i}$, for each filter $i$ by solving the following equation with a least-square fit

$m_{\mathrm{s}}=m_{0}+k_{i} \cdot X+z_{i}$

where $m$ is the magnitude of the star and $X$ is the airmass. The subscripts $s, 0$ and $i$ designates the standard magnitude, the observed magnitude and the filter, respectively. With preliminary zeropoints and extinction coefficients we then solved for the colour term, $a_{i}$, using a least-square fit

$m_{\mathrm{s}}=m_{0}+a_{i} \cdot(v-y)_{\mathrm{s}}+z_{i}^{\prime}$

where $z_{i}{ }^{\prime}$ is the residual from the linear fit. Note that here we use the data for both nights in order to make use of the colours for all standard stars. With preliminary colour terms we can now solve the full equation to obtain better estimates of $z_{i}$ and $k_{i}$

$m_{\mathrm{s}}=m_{0}+a_{i} \cdot(v-y)_{\mathrm{s}}+k_{i}{ }^{\prime \prime} \cdot X+z_{i}^{\prime \prime}$

with these better estimates, Eq. (2) is again solved and Eqs. (2) and (3) are then iterated until convergence in $a, z$ and $k$ is achieved. Typically convergence is reached within 6 to 7 iterations. The final zeropoint is given by $z^{\prime \prime}$ in Eq. (3) plus the residual zeropoint from Eq. (2) from the last iteration. The extinction coefficients, colour terms, zeropoints and correlation coefficients between the uncertainty in $z$ and $k, \rho_{z k}$, for each filter and night are listed in Table 3 . We note that the uncertainty in zeropoint and extinction coefficient are strongly anti-correlated.

The $z, k$ and $a$ obtained were used to transform our observations onto the standard system of Olsen (1993). The uncertainty for $z, k$ and $a$, and the correlation coefficient $\rho_{z k}$ were used to calculate the errors in the magnitudes, Sect. 2.1.4.

In Fig. 2 we show the residuals between our photometry and the standard values from Schuster \& Nissen (1988) and Olsen (1993) as a function of our calibrated $(v-y)$ colour. We note that there are no trends with colour.

\subsubsection{Photometry of the stars in the science images}

Instead of co-adding the science images, we did aperture photometry on each of the images separately. Co-adding the images would be difficult since the seeing varied from night to night and because of the necessity to apply the extinction correction for each night separately. As described later in this section the final flux for each star in each filter was calculated using a weightedmean of the individual measurements. This enables us to do a more detailed study of the night to night quality. As a quality 
Table 3. Coefficients for Eq. (3).

\begin{tabular}{ccccc}
\hline \hline Night & $k_{y}$ & $z_{y}$ & $a_{y}$ & $\rho_{z k}$ \\
\hline 14 April 2007 & $-0.155 \pm 0.021$ & $23.009 \pm 0.031$ & $0.016 \pm 0.005$ & -0.92 \\
15 April 2007 & $-0.142 \pm 0.016$ & $22.989 \pm 0.023$ & $0.016 \pm 0.005$ & -0.96 \\
\hline Night & $k_{b}$ & $z_{b}$ & $a_{b}$ & $\rho_{z k}$ \\
\hline 14 April 2007 & $-0.240 \pm 0.031$ & $23.337 \pm 0.043$ & $0.009 \pm 0.005$ & -0.97 \\
15 April 2007 & $-0.210 \pm 0.036$ & $23.297 \pm 0.050$ & $0.009 \pm 0.005$ & -0.96 \\
\hline Night & $k_{v}$ & $z_{v}$ & $a_{v}$ & $\rho_{z k}$ \\
\hline 14 April 2007 & $-0.349 \pm 0.023$ & $23.051 \pm 0.034$ & $0.050 \pm 0.006$ & -0.98 \\
15 April 2007 & $-0.353 \pm 0.023$ & $23.048 \pm 0.032$ & $0.050 \pm 0.006$ & -0.96 \\
\hline Night & $k_{u}$ & $z_{u}$ & $a_{u}$ & $\rho_{z k}$ \\
\hline 14 April 2007 & $-0.582 \pm 0.036$ & $23.131 \pm 0.052$ & $0.065 \pm 0.007$ & -0.98 \\
15 April 2007 & $-0.569 \pm 0.013$ & $23.117 \pm 0.021$ & $0.065 \pm 0.007$ & -0.96 \\
\hline
\end{tabular}

Columns 2 to 4 list the airmass extinction coefficients, $k_{i}$, zeropoints, $z_{i}$ and colour coefficients, $a_{i}$, for each filter as indicated with uncertainties $\sigma_{k}$, $\sigma_{z}$ and $\sigma_{a}$ respectively. Column 5 lists the correlation coefficient between the uncertainty in extinction coefficient, $\sigma_{k}$, and uncertainty in zeropoint, $\sigma_{z}$. This coefficient is denoted by $\rho_{z k}$.

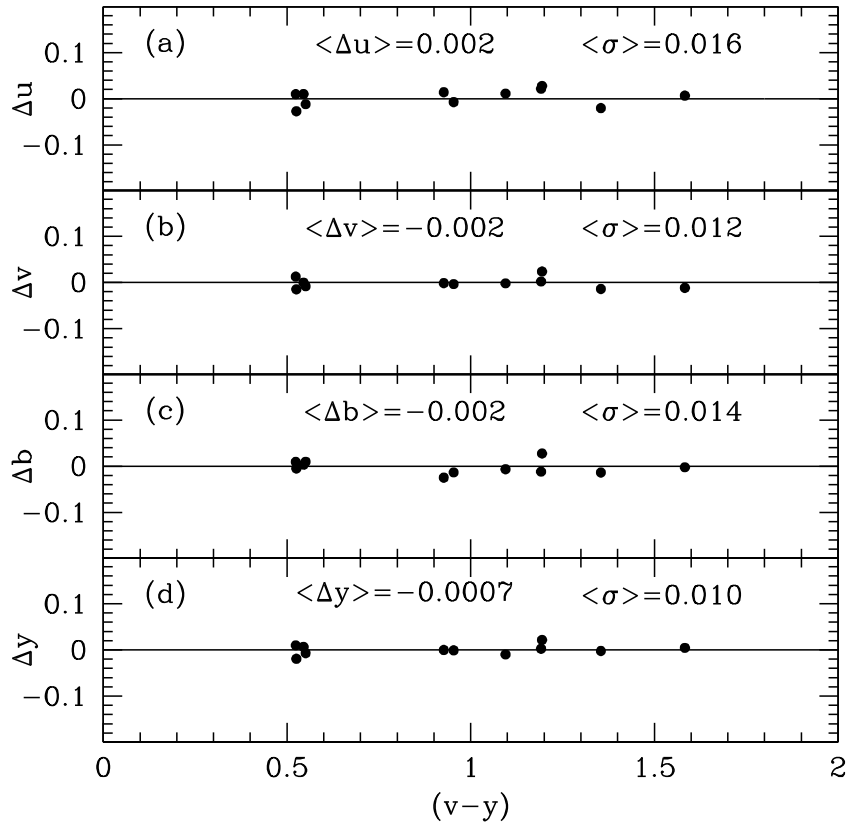

Fig. 2. The residuals for the standard stars as a function of our final $(v-y)$ colour. The residuals are given in the sense "our observed value" "the standard value". Mean differences and $\sigma$ as indicated.

check we compared the flux for the brightest targets for each night with a mean flux, calculated for each object for all nights, to see if any of the images deviated in flux. None of the images deviated. From this we draw the conclusion that the calibration was consistent throughout the entire observing run.

Coordinate lists for the images were created using the task DAOFIND in the APPHOT package in IRAF. To establish the coordinate lists we used the best $y$ image, since the stars are brightest in this filter. We then used this catalogue of coordinates for all the other images. We used the aperture photometry task PHOT, within the APPHOT package, to measure the flux for all objects on the images.

Aperture correction. When doing photometry on the science images we used a fixed aperture of 5 pixels. Applying a curveof-growth we obtained, for each individual image, the aperture correction out to $4 \times F W H M$ of the $p s f$. The aperture corrections were based on measurements of many bright isolated stars,
Table 4. Aperture corrections.

\begin{tabular}{lccc}
\hline \hline File name & Filter & CCD chip & Aperture correction \\
\hline $\mathrm{r} 556732$ & $y$ & $1 / 2 / 3 / 4$ & $1.265 / 1.266 / 1.278 / 1.266$ \\
$\mathrm{r} 556737$ & $b$ & $1 / 2 / 3 / 4$ & $1.273 / 1.262 / 1.274 / 1.256$ \\
$\mathrm{r} 556748$ & $v$ & $1 / 2 / 3 / 4$ & $1.195 / 1.191 / 1.164 / 1.168$ \\
$\mathrm{r} 556754$ & $u$ & $1 / 2 / 3 / 4$ & $1.171 / 1.161 / 1.135 / 1.148$ \\
$\mathrm{r} 556882$ & $b$ & $1 / 2 / 3 / 4$ & $1.269 / 1.264 / 1.259 / 1.240$ \\
$\mathrm{r} 556887$ & $y$ & $1 / 2 / 3 / 4$ & $1.162 / 1.166 / 1.162 / 1.155$ \\
$\mathrm{r} 556893$ & $v$ & $1 / 2 / 3 / 4$ & $1.268 / 1.264 / 1.223 / 1.238$ \\
$\mathrm{r} 556898$ & $u$ & $1 / 2 / 3 / 4$ & $1.378 / 1.366 / 1.312 / 1.343$ \\
$\mathrm{r} 556899$ & $u$ & $1 / 2 / 3 / 4$ & $1.384 / 1.362 / 1.316 / 1.352$ \\
$\mathrm{r} 556997$ & $y$ & $1 / 2 / 3 / 4$ & $1.254 / 1.261 / 1.254 / 1.244$ \\
$\mathrm{r} 557004$ & $b$ & $1 / 2 / 3 / 4$ & $1.212 / 1.203 / 1.204 / 1.188$ \\
$\mathrm{r} 557009$ & $v$ & $1 / 2 / 3 / 4$ & $1.382 / 1.382 / 1.336 / 1.356$ \\
$\mathrm{r} 557018$ & $u$ & $1 / 2 / 3 / 4$ & $1.209 / 1.198 / 1.160 / 1.185$ \\
$\mathrm{r} 557019$ & $u$ & $1 / 2 / 3 / 4$ & $1.335 / 1.322 / 1.282 / 1.308$ \\
\hline
\end{tabular}

Column 1 lists the file name for the image as named by the observing and archiving system on the Isaac Newton Telescope. Column 2 lists the filter. Column 3 lists the CCD chip number and Col. 4 the aperture correction for each CCD in the same order as in Col. 3 .

Table 5. Summary of the spectroscopic observations with FLAMES.

\begin{tabular}{cc}
\hline \hline Date & Exp. time [min] \\
\hline 15 April 2007 & 45 \\
15 April 2007 & 45 \\
10 May 2007 & 45 \\
17 May 2007 & 40 \\
21 June 2007 & 40 \\
21 June 2007 & 40 \\
21 June 2007 & 40 \\
\hline Total Exp. Time & 295 \\
\hline
\end{tabular}

Column 1 lists the date of observation and Col. 2 the exposure time.

typically 20 stars per CCD. The aperture corrections were done in flux-space and are listed in Table 4.

Final magnitudes. Initial magnitudes were calculated for each object and night for every image and calibrated for the airmass extinction and zeropoint using Eq. (2), but this time with subscript $s$ as our calibrated magnitude and, as before, 0 as the observed magnitude, with coefficients from Table 3. Since the first night did not give us reliable standard star photometry, and we thus have no calibration for that night, we normalized the 
magnitudes from that night to the mean of the magnitudes for the two following nights.

For all three nights, erroneous measurements returned from PHOT for the individual exposures were removed from the data set (i.e. the measurements for which sier, cier and pier $\neq 0$, which are the error in sky fitting, centering algorithm and photometry, respectively). The flux was then calculated for each star. The final flux, $\bar{F}$, was obtained by using a weightedmean flux where the photometric errors returned from PHOT (merr) were used as weights. The expression for the final flux is thus

$\bar{F}=\frac{\sum_{j=1}^{n} f_{j} / \sigma_{j}^{2}}{\sum_{j=1}^{n} 1 / \sigma_{j}^{2}}$

where the subscript $j$ is the exposure, $n$ is the total number of exposures, $f_{j}$ is the flux of the individual exposure and $\sigma_{j}$ the error (merr). These mean fluxes were then converted back to magnitudes and the colour terms were applied to get the final magnitudes. By definition, $y \equiv V$ (e.g. Olsen 1983), and we will henceforth use $V$ instead of $y$ in figures and discussions.

\subsubsection{Photometric errors}

The errors in magnitude for the Strömgren photometry were calculated using a Monte Carlo Simulation, taking into account merr, the uncertainty in zeropoint, extinction coefficient and colour term and the strong anti-correlation between the uncertainty in zeropoint and extinction coefficient.

We did this in the following way. For each object, a new magnitude was calculated

$m_{i}=m_{0, i}+\Delta m$

where $m_{0}$ is the observed magnitude in filter $i$ and $\Delta m$ is a random number, drawn from a normal distribution with a mean of 0 and a variance of $\sigma_{m}^{2}$, where $\sigma_{m}$ is the error in the magnitude as returned from the PHOT task (merr). Additionally, for each object, a new zeropoint and extinction coefficient were calculated

$z_{i}=z_{0, i}+\Delta z$

$k_{i}=k_{0, i}+\Delta k$

where $z_{0, i}$ and $k_{0, i}$ are the zeropoint and extinction coefficient for filter $i$ used in the calibration (see Sect. 2.1.2) and $\Delta z$ is a random number, drawn from a normal distribution with a mean of 0 and a variance of $\sigma_{z}^{2}$, see Table 3. Since $\sigma_{z}$ and $\sigma_{k}$ are strongly anti-correlated, we take into account the correlation coefficient from the calibration when calculating the random number $\Delta k$ (see Sect. 2.1.2 and Table 3). Finally, a new colour term was calculated

$a_{i}=a_{0, i}+\Delta a$

where $a_{0, i}$ is the calculated colour term for filter $i$ and $\Delta a$ is a random number, drawn from a normal distribution with a mean of 0 and a variance of $\sigma_{a}^{2}$.

This process was then repeated 2000 times, generating a new set of $u, v, b$ and $y$ magnitudes in each iteration. As the final error for each magnitude we adopt the standard deviation of the magnitudes from the Monte Carlo simulation. This is calculated as follows

$\sigma_{\bar{m}}=\sqrt{\frac{1}{n-1} \sum_{j=1}^{n}\left(m_{j}-\bar{m}\right)^{2}}$
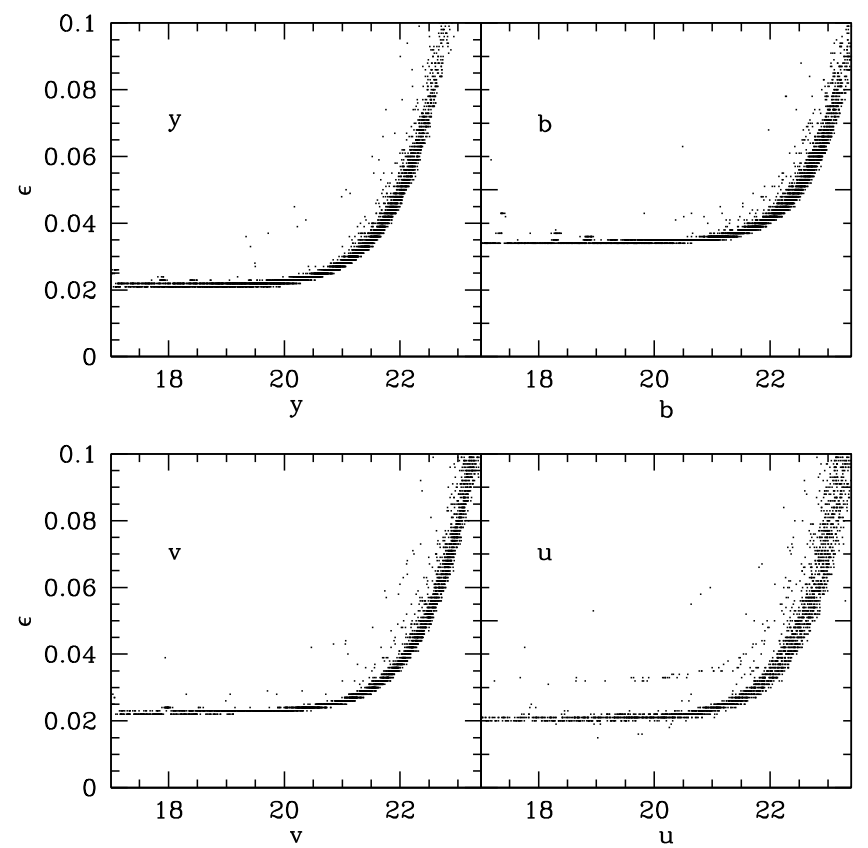

Fig. 3. Errors in the photometry for $y, b, v$ and $u$, as indicated. The calculation of the errors is described in Sect. 2.1.4. The few stars with anomalously high errors at a given magnitude are also discussed there.

where the subscript $j$ is the Monte Carlo iteration number, and $\bar{m}$ the mean magnitude of the distribution.

Figure 3 presents our final photometric errors, $\epsilon$. The baselevel error of $\sim 0.02 \mathrm{mag}$ ( 0.035 for the $b$ filter) is due mainly to the errors in the photometric calibration, see Table 3 and the discussion above. The profile of the errors, i.e. increasing error with decreasing magnitude, is dominated by merr (i.e. photon statistics). Some stars show a larger $\epsilon$ than the majority of stars at that magnitude. This is most obvious in the $u$ filter and is a statistical feature caused by the number of exposures that are included for that star. The $u$ filter suffers most from this since stars are generally a lot fainter in this filter, and therefore more measurements are rejected due to errors in sky fitting, centering and photometry. For example, the stars in $u$ with a larger $\epsilon$ for a given magnitude have three or fewer individual measurements while the stars in the main trend all have five measurements. The higher error in $b$ is caused by the higher uncertainty for the zeropoint in $b$ (compare Table 3 ).

\subsubsection{Stellar classification using SExtractor}

Contamination by background galaxies is a concern for our observations. As our images are uncrowded we can reach faint magnitudes with relative ease using aperture photometry. However, by using aperture photometry we have no information about the shape of the objects. In order to sort the stars from background galaxies we used SExtractor v2.5. SExtractor uses a tunable neural network trained on realistic simulated images to separate galaxies from stars in moderately crowded images (Bertin \& Arnouts 1996). The SExtractor output, which is of interest to us, is the stellarity index, sclass; sclass takes on values between 0 and 1 , where 0 indicates a galaxy and 1 a star. Following Bertin \& Arnouts (1996), objects with a stellarity index greater than or equal to 0.5 were identified as stars.

As can be seen in Fig. 4, SExtactor clearly removes galaxies at bright magnitudes but its ability to distinguish stars from 


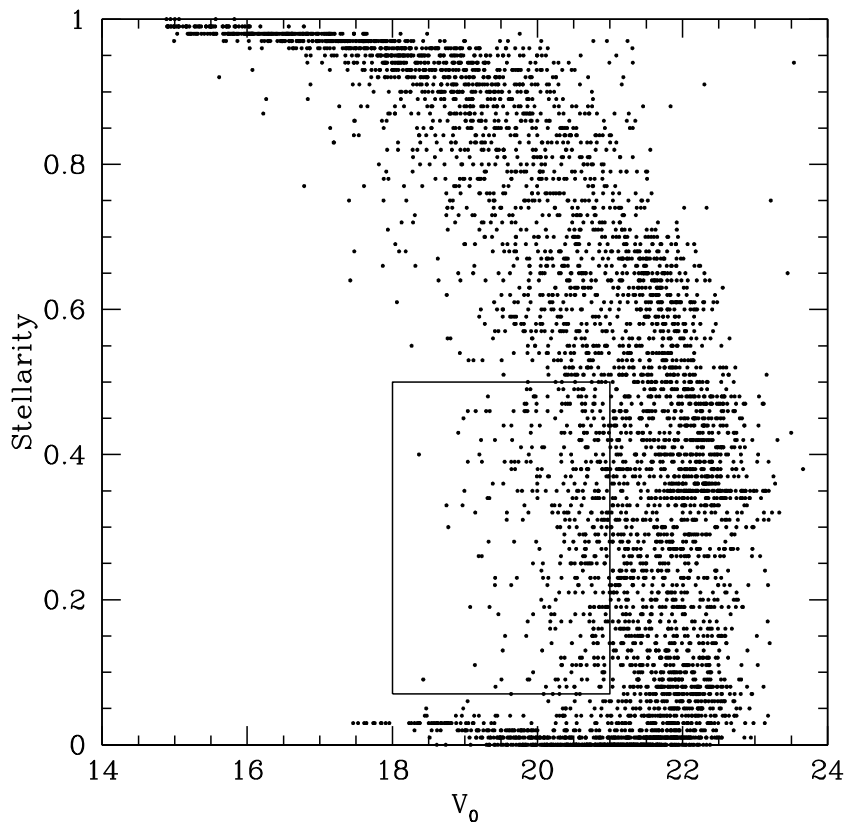

Fig. 4. SExtractor stellarity index (sclass) as a function of $V_{0}$. A star takes on values close to 1 and galaxies values close to 0 . The rectangle indicates the area where we investigated each object individually (see Sect. 2.1.5).

galaxies diminishes at fainter magnitudes where the $S / N$ is lower. During our membership analysis (see Sect. 4.2) we investigated objects in a stellarity index range $0.07<$ sclass $<0.5$ and magnitude range $18<V_{0}<21$ in order to make sure that we did not exclude any objects of interest based on the stellarity index alone. This area is marked with a solid line in Fig. 4. We found two objects of interest, INT 34489 and INT43290 (see Sect. 4.2 for a discussion of these two objects).

\subsection{Spectroscopy}

Our spectroscopy was carried out using the multiobject spectrograph Fibre Large Array Multi Element Spectrograph (FLAMES) at the Very Large Telescope (VLT) on Paranal. Operated in Medusa fibre mode, this instrument allows for the observation of up to 130 targets at the same time (Pasquini et al. 2002). Figure 1 shows the fibre positions on the sky for the stellar targets. 21 additional fibres were dedicated to observing blank sky. We used the GIRAFFE/L8 grating, which provides a nominal spectral resolution of $R \sim 6500$ and a wavelength coverage from $821 \mathrm{~nm}$ to $940 \mathrm{~nm}$, centred on the Ca II IR triplet lines in the spectral region around $860 \mathrm{~nm}$.

\subsubsection{Reduction of spectroscopic observations}

Initially, the FLAMES observations were reduced with the standard GIRAFFE pipeline version 2.2 (Blecha et al. 2000). This version of the pipeline, however, was not able to reduce a persistent glow on the CCD (Lind et al. 2008). This glow then created an extra background that increases towards the red. The effect was very large and would have affected the equivalent width measurements. Fortunately, during our work with these spectra a beta-version of the next version of the pipeline became available. The data were thus re-reduced with the GIRAFFE pipeline, version 2.5. This pipeline provides bias subtraction, flat fielding, dark-current subtraction, and accurate wavelength calibration from a ThAr lamp. It also solved the issue of the CCD glow.

\subsubsection{Spectroscopic measurements}

The 21 sky spectra were combined and subtracted from the object spectra with the task SKYSUB in the SPECRED package in IRAF. Figure 5 gives an example of the sky-subtraction process.

Finally, the object spectra from the individual frames were Doppler-shifted to the heliocentric rest frame and mediancombined into the final one-dimensional spectrum. When combining the object spectra we used an average sigma clipping algorithm, rejecting measurements deviating by more than $3 \sigma$, in order to remove cosmic rays.

Radial velocities were determined by a Fourier crosscorrelation of the combined spectra against a synthetic template spectrum using the IRAF task FXCOR. The template consisted of three Gaussian absorption lines at the positions of the Ca II IR triplet lines, with equivalent widths $(W)$ representative for red giant stars. The radial velocities were determined from a Gaussian fit to the strongest correlation peak within a $300 \mathrm{~km} \mathrm{~s}^{-1}$ window. The uncertainty in the measurement of the radial velocity was returned by FXCOR and is based on the Tonry-Davis $R$-value (Tonry \& Davis 1979, see Figs. 7a and b).

During the Fourier cross-correlation process we performed an ocular inspection of the quality of the spectra. Spectra of objects fainter than $V_{0} \sim 21.3$ showed no clearly visible Ca II IR triplet lines and were thus removed from the sample. The $S / N$ for these spectra was typically $\sim 4$ or less.

The equivalent widths, $W$, for the $\mathrm{Ca}$ II IR triplet lines were measured by fitting a Gaussian profile (Cole et al. 2004) to each of the three lines using the IRAF task SPLOT. From an ocular inspection of the spectra we found that the Gaussian profile fitted the Ca II triplet lines better than a Voigt profile.

\subsection{Interstellar reddening}

We corrected the photometric magnitudes for interstellar extinction using the dust maps by Schlegel et al. (1998). This gives $E(B-V)=0.062$, in agreement with Koch et al. (2008b).

Coleman et al. (2007) used a reddening of $E(B-V)=0.055$ with an uncertainty of 0.005 that represents the variation in reddening over the Large Binocular Telescope $23^{\prime} \times 23^{\prime}$ field. In Sect. 8.1 we investigate how different values of $E(B-V)$ affect the estimated metallicities for the stars.

We used the Schlegel et al. (1998) relations to translate these extinction values into the Strömgren system. De-reddened magnitudes, colours and Strömgren indices will henceforth have the subscript 0 .

\section{Results}

\subsection{Colour-magnitude diagram in the direction of the Hercules dSph galaxy}

Figure 6 presents our colour-magnitude diagrams in the direction towards the Hercules dSph galaxy. The horizontal branch (HB) is seen at $V_{0} \approx 21.2$ in Fig. 6a. A large population of foreground stars can also be seen with a cut-off at $(b-y)_{0} \approx 0.3$, associated with the blue limit of the turnoff stars in the Milky Way disk and halo. The RGB of the Hercules dSph galaxy cannot easily be seen due to the heavy contamination by foreground dwarf stars. 


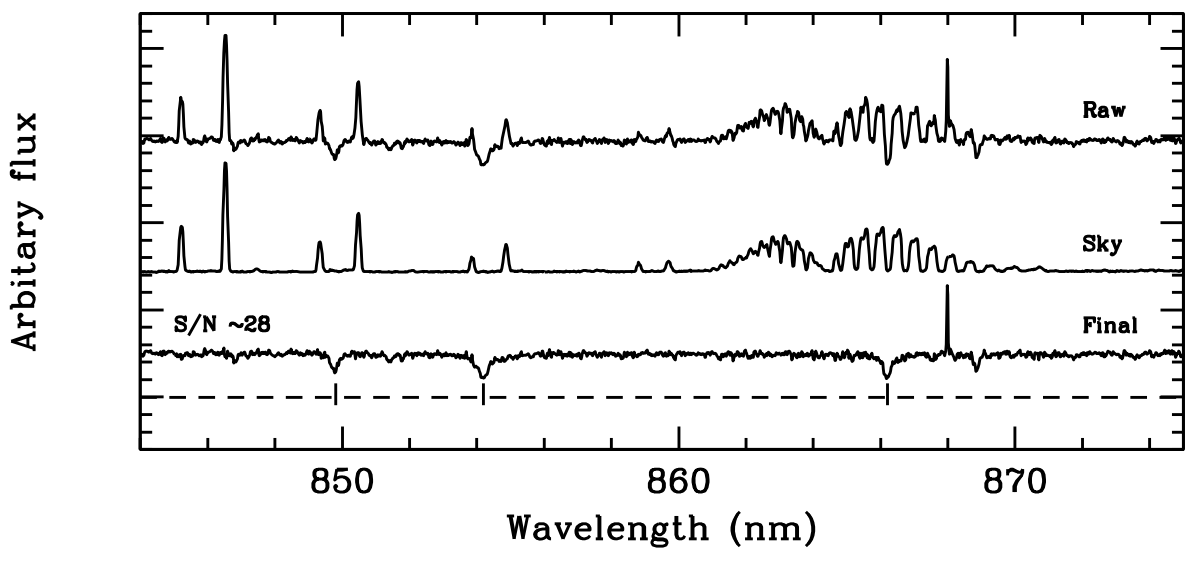

Fig. 5. Example showing the subtraction of skyemission lines from the stellar spectra. At the top we show a raw, one-dimensional, spectrum for one of the brightest stars in our sample, $V=18.4$. Below that spectrum is the final spectrum of the sky constructed through the combination of all 21 sky fibres. At the bottom we show the stellar spectrum once the sky has been removed. The $S / N$ for the final stellar spectrum is 28 . The rms in the sky spectrum is $\sim 1.1$ counts and $\sim 9.8$ counts in the stellar spectrum. The three Ca II IR triplet lines are indicated by vertical lines in the bottom spectrum. The dashed line indicates the position of zero intensity for the final spectrum.

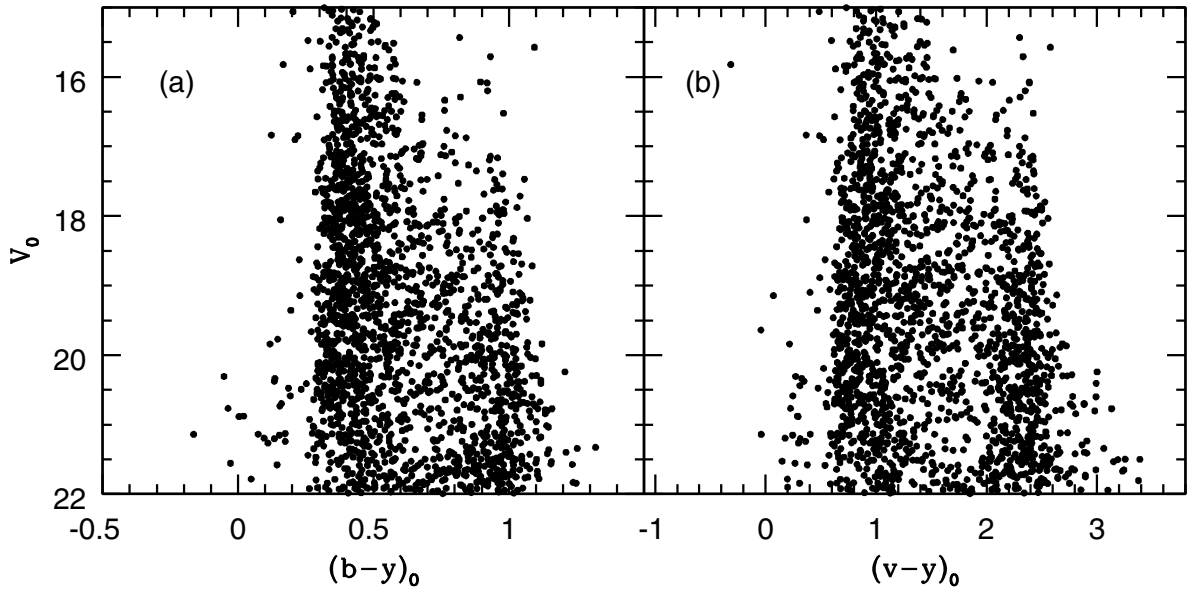

Fig. 6. Colour-magnitude diagram centred on the Hercules dSph galaxy. a) $V_{0}$ vs. $(b-y)_{0}$. b) $V_{0}$ vs. $(v-y)_{0}$. Only objects with a stellarity index sclass $\geq 0.5$ are shown (see Sect. 2.1.5).

\subsection{Radial velocities}

Figure 7a shows the stellarity index as a function of the derived radial velocity for the objects observed with FLAMES. Objects with a stellarity index less than 0.5 are excluded from the sample (see Sect. 2.1.5). We investigated the objects with a stellarity index less than 0.5 and a velocity close to the systemic velocity and found that all of these objects are fainter than $V_{0}=21$ except three objects, INT 43290, INT 40586 and INT 41659. INT 43290 lies on top of a background galaxy and is therefore contaminated. INT 40586 and INT 41659 have a questionable surface distribution on the CCD. We flag them as blends and possible double-star systems and identify them in Fig. 7b.

Figure $7 \mathrm{~b}$ shows the magnitude of the stars vs. the derived radial velocities. The error-bars on the radial velocities are from the Tonry-Davis $R$-value (Tonry \& Davis 1979) estimates. As expected, errors in the velocities correlate with magnitude such that fainter stars have larger errors.

In Fig. 8 we compare the distribution of our radial velocities in the direction of the Hercules dSph galaxy with the predictions of the Besançon model (Robin et al. 2003) with the same area on the sky as that covered by our observations. The colour and magnitude range for the distribution of stars from the Besançon model is the same as spanned by the FLAMES targets. As can be seen, also in this respect the Hercules dSph galaxy suffers from heavy foreground contamination. We note that the observed velocity distribution is not centred on the field star distribution predicted by the model, but is shifted by about $-25 \mathrm{~km} \mathrm{~s}^{-1}$. Moreover, disregarding the peak created by the Hercules members, the observed distribution is narrower than the model prediction. This may point to some problem with the model; however, a full investigation of the origin of this difference is beyond the scope of the present study.

\section{Finding the giant and horizontal branch stars in the Hercules dSph galaxy}

\subsection{The ability of the Strömgren $c_{1}$ index to identify $R G B$ stars}

As Figs. 6 and 8 show, the line of sight towards the Hercules $\mathrm{dSph}$ galaxy is heavily contaminated with foreground stars, making it impossible to determine membership from the Colourmagnitude diagram alone. Even when radial velocities are added the selection remains uncertain, because the mean velocity of the Hercules dSph galaxy coincides with the velocity of the (thick) disk.

The $c_{1}$ index in the Strömgren system gives us the ability to disentangle the RGB and $\mathrm{HB}$ stars in a dSph galaxy from the foreground dwarf stars. The $c_{1}$ index is a measure of the Balmer discontinuity in a stellar spectrum and is defined as

$c_{1}=(u-v)-(v-b)$.

The strength of the Balmer discontinuity depends on the evolutionary stage of the star. Stars in a plot of $c_{1,0}$ vs. $(b-y)_{0}$ will therefore occupy different regions depending on their evolutionary stage. Figure 9 shows which regions are occupied by stars at different evolutionary stages. This classification is adopted from Schuster et al. (2004). We also show the region occupied by the RGB stars in the Draco dSph galaxy (Faria et al. 2007).

Schuster et al. (2004) were mainly concerned with high velocity dwarf stars and to a lesser extent interested in the redder 

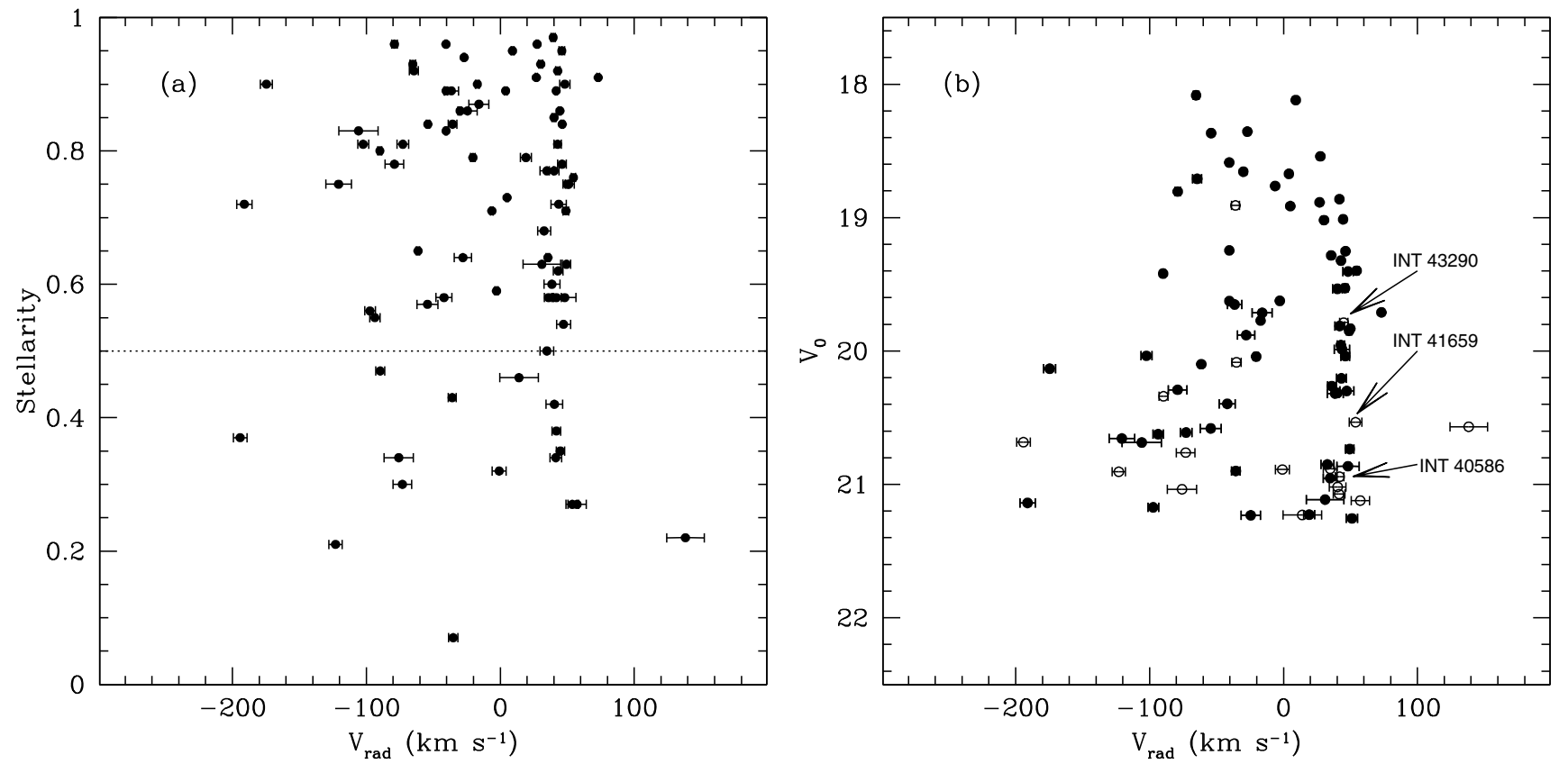

Fig. 7. a) SExtractor stellarity index vs. radial velocity for the objects observed with FLAMES. The error-bars represent the error in $V_{\text {rad }}$ as returned by the task FXCOR. The dotted line indicates stellarity index 0.5 . b) $V_{0}$ vs. radial velocity. $\bullet$ indicates objects with a stellarity index greater than 0.5 . The error-bars represent the error in $V_{\text {rad }}$ as returned by the task FXCOR. $\circ$ indicates objects with a stellarity index lower than 0.5 . Three stars are identified with their INT numbers. These stars are discussed in Sects. 3.2 and 4.2.

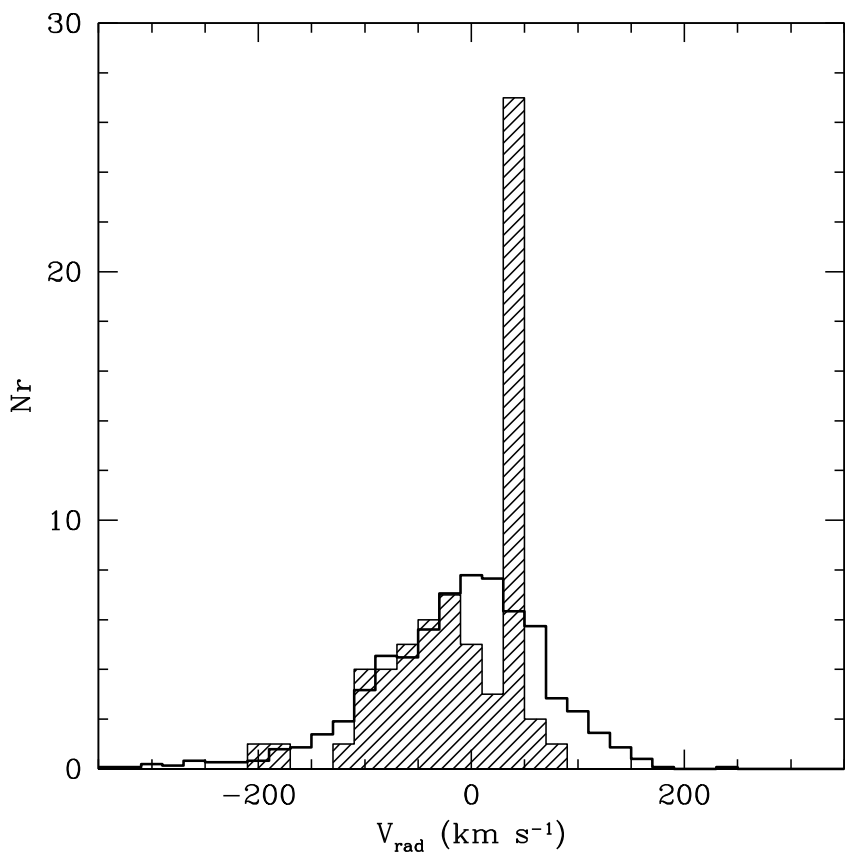

Fig. 8. Distributions of radial velocities. The solid histogram shows the distribution of velocities from a Besançon model in the direction of the Hercules dSph galaxy. The shaded histogram shows the distribution of radial velocities for our FLAMES observations. Only objects with sclass $>0.5$ are included (see Sect. 2.1.5). Note that the area of the histogram based on the Besançon model has been normalized to cover the same area as the FLAMES histogram.

dwarf and RGB stars. As the high-velocity halo stars that they studied tend to be fairly blue we will use tracings for dwarf stars from Árnadóttir et al. (in preparation), and isochrones for RGB stars by VandenBerg et al. (2006) with colour transformations by Clem et al. (2004), in the $c_{1,0}$ vs. $(b-y)_{0}$ diagram to define

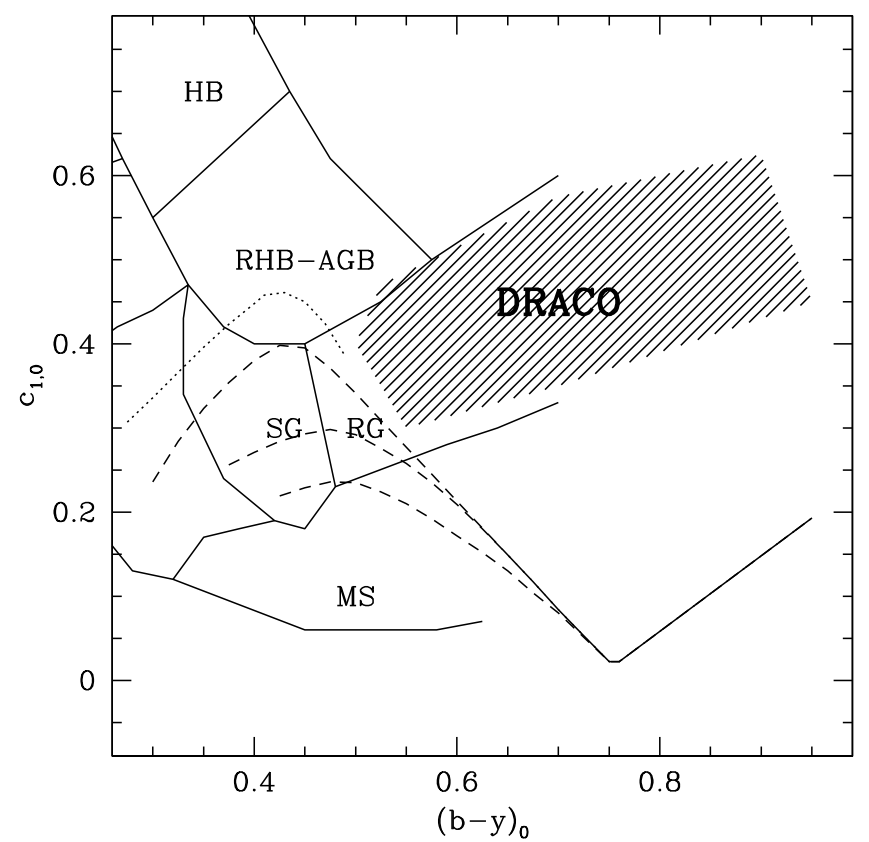

Fig. 9. $c_{1,0}$ vs. $(b-y)_{0}$ diagram with regions occupied by stars at various evolutionary stages as defined in Schuster et al. (2004). MS: the main sequence; SG: sub-giant stars; RG: red giant stars; RHB-AGB: the red horizontal-branch-asymptotic-giant-branch transition; HB: the horizontal branch. The shaded region marked DRACO indicates the region occupied by the RGB stars in the Draco dSph galaxy (Faria et al. 2007). The dashed lines indicate dwarf star sequences for different metallicities, $[\mathrm{Fe} / \mathrm{H}]=0.45,-0.05$ and -1 from top to bottom and the dotted line marks the upper envelope for dwarf stars (all lines from Árnadóttir et al., in preparation).

the dwarf and giant star regions also in the red. The dwarf sequences in Árnadóttir et al. (in preparation) provide an extension of the preliminary dwarf relation from Olsen (1984). The major 


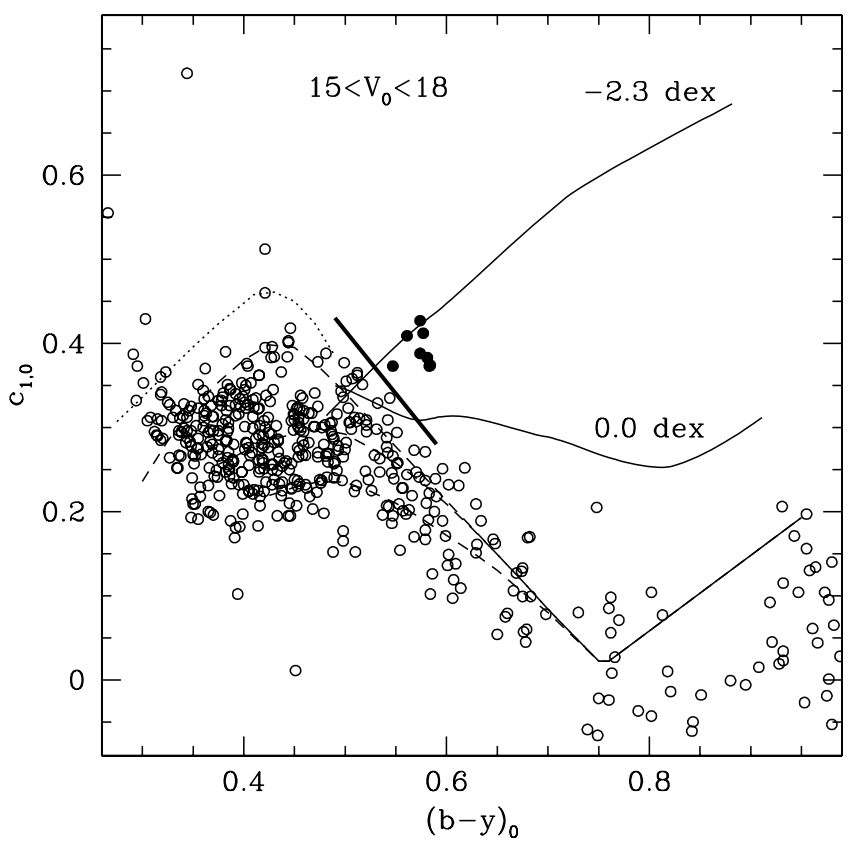

Fig. 10. $c_{1,0}$ vs. $(b-y)_{0}$ for stars, $\circ$, in the direction of the Hercules dSph galaxy. Only stars in the magnitude range $15<V_{0}<18$ are shown. mark stars that fall within the RGB region as defined by the isochrones by VandenBerg et al. (2006) with colour transformations by Clem et al. (2004). Metallicities as indicated. The thin dashed lines indicates dwarf star sequences for different metallicities, $[\mathrm{Fe} / \mathrm{H}]=0.45,-0.05$ and -1 top to bottom and the dotted line marks the upper envelope for dwarf stars (all lines from Árnadóttir et al., in preparation). The thick line marks the empirically determined limit for the foreground contamination (see Sect. 4.1).

difference between the preliminary relation by Olsen (1984) and the new relations is that the new relations are functions of metallicity. In Fig. 9 we show dwarf sequences for three different metallicities. These sequences, in accordance with Olsen (1984), trace the lower envelope for the dwarf stars (for $(b-y)_{0}$ less than about 0.55$)$. Note that for redder colours $\left((b-y)_{0}\right.$ larger than about 0.55$)$ all dwarf sequences merge and form a single line that traces the mean values of the colours. By studying dwarf stars from Olsen $(1993,1994 \mathrm{a}, \mathrm{b})$ in a $c_{1,0}$ vs. $(b-y)_{0}$ diagram it is possible to define an upper envelope for the region occupied by foreground dwarf stars. Árnadóttir et al. (in preparation) define such an upper envelope. We include this in our plots henceforth.

To define the RGB region we use two isochrones with $[\mathrm{Fe} / \mathrm{H}]=-2.3$ and $[\mathrm{Fe} / \mathrm{H}]=0.0$ by VandenBerg et al. (2006) and colour transformations by Clem et al. (2004), see Fig. 10.

As can be seen from Fig. 10, for giant stars, the $c_{1}$ index has a clear metallicity dependence. This is more prononounced for the reddest colours (i.e. the tip of the RGB). However, in spite of this, this index still provides a strong discriminant between giant and dwarf stars for cooler stars. Note that the dwarf sequence is not metallicity dependent at these colours.

The blue limit for membership determination. Since in the $c_{1,0}$ vs. $(b-y)_{0}$ plane the dwarf and RGB stellar sequences converge around $(b-y)_{0} \sim 0.5$ we need to identify a blue limit for stars that we identify as RGB stars. In Fig. 10 we show the $c_{1,0}$ vs. $(b-y)_{0}$ for stars in the magnitude range $15<V_{0}<18$. Stars fainter than $V_{0}=15$ are not saturated on the images and, based on our membership-determination in the next section, this magnitude range is bright enough not to contain any RGB stars in the

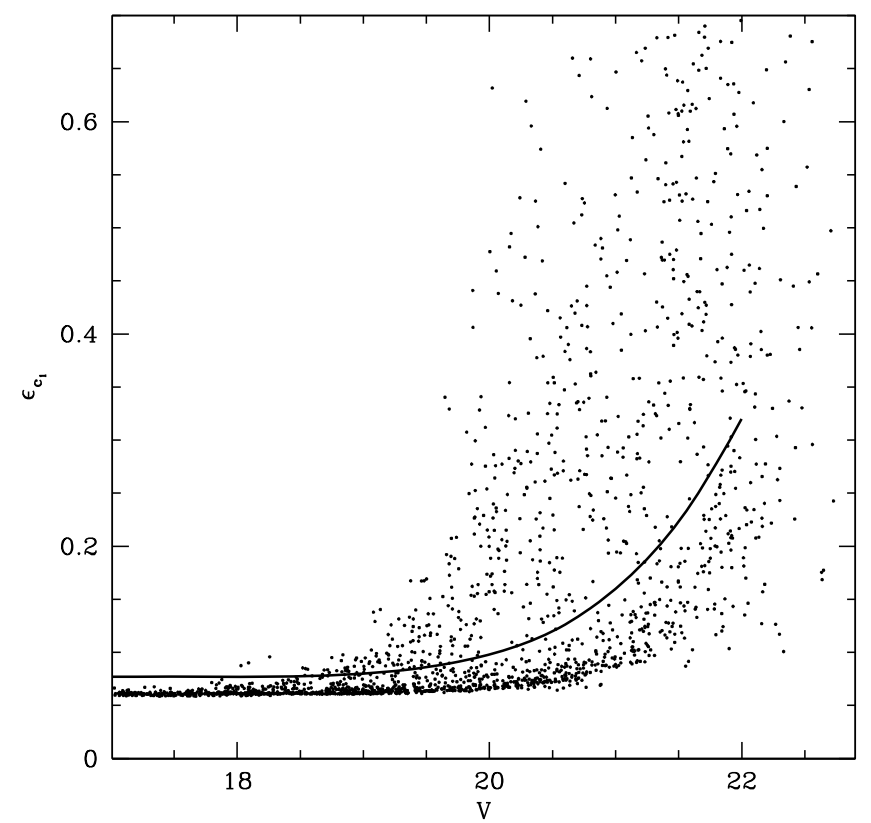

Fig. 11. Errors in the Strömgren colour index $c_{1}$ (see Sect. 4.1). The solid line indicates a spline function fitted to fall just above the trend with smaller errors. This line is used in Sect. 4.2 and Figs. 12 and 13 where we define the RGB stars.

Hercules dSph galaxy. We define a line that follows the upper envelope of observed dwarf stars to separate the RGB stars from the dwarf stars in order to safely exclude any dwarf stars. This line is somewhat higher in $c_{1,0}$ at a given $(b-y)_{0}$ than the tracing from Árnadóttir et al. (in preparation). Our selection of RGB stars thus has a blue limit that is somewhat colour-dependent. No object bluer than this limit will be considered as an RGB star since they have a high probability of belonging to the foreground dwarf contamination.

In Fig. 10 we see 8 stars within the RGB region. A Besançon model in the direction of the Hercules dSph galaxy gives 15 RGB stars in the given magnitude and colour range. The conclusion is therefore that these stars are most likely foreground RGB stars belonging to the thick disc of the Milky Way.

\subsection{Membership based on Strömgren photometry.}

We study the $c_{1,0}$ vs. $(b-y)_{0}$ in magnitude bins rather than the entire sample of all stars all at once. With this approach the RGB region is easier to track. Figure 11 shows the errors in $c_{1,0}$ as a function of magnitude. We note the large errors in $c_{1}$ for fainter stars. These errors are due to the higher errors in $u$ at any given magnitude. Note that in terms of errors (see Fig. 3), $V=20$ corresponds to $u \sim 22.5$ for our RGB stars. In order to exclude stars with large errors, we reject all stars with errors higher than the median error, plus $0.015 \mathrm{mag}$, at a given magnitude (as indicated by the solid line in Fig. 11). We will now use this function in our classification of members and potential members of the Hercules dSph galaxy.

Figure 12a shows a clear and simple separation between the foreground dwarf stars and the four stars that fall in the RGB region. These four RGB stars all have errors in $c_{1,0}$ less than the line fitted in Fig. 11. Figures $12 b-d$ show the three remaining magnitude bins. We also note that 9 stars fall within the RHBAGB region, they are discussed in Sect. 4.3. 


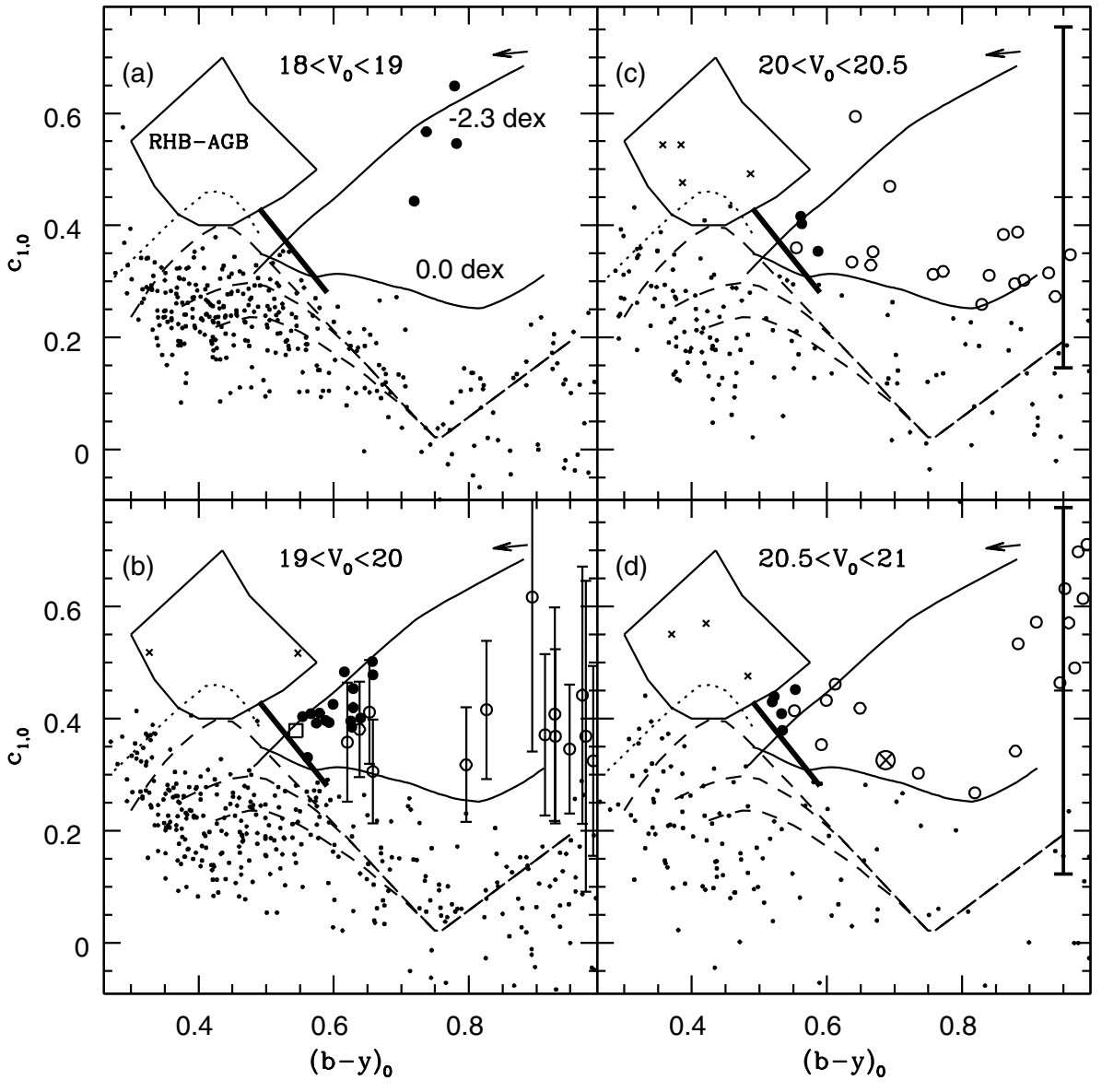

Fig. 12. $c_{1,0}$ vs. $(b-y)_{0}$ for stars in the direction of the Hercules dSph galaxy. The range of magnitude is indicated at the top of each panel. Dots are stars within the given magnitude range. $\bullet$ are stars that fall within the RGB region and have errors less than the line defined in Fig. 11. These are members based on their evolutionary stage. $\circ$ are stars that fall within the RGB and have errors larger than the line defined in Fig. 11. The star marked with $\otimes$ in $\mathbf{d}$ ) is flagged as a non-member due to its position in the $\epsilon_{c_{1}}$ vs. $(b-y)_{0}$ plane, see Fig. 13. The star marked with a $\square$ in c) is a foreground RGB star (see Sect. 4.2). The errorbars in b) represent the error in $c_{1}$ and are only displayed for stars that fall above the line defined in Fig. 11. The thick error-bars to the right in c) and d) represent the mean error in $c_{1}$ for stars that fall above the line defined in Fig. 11 (०). $\times$ marks stars that fall in the RHB-AGB region (see Sect. 4.3). The arrow in the top right corner in each panel indicates the magnitude and direction of the de-reddening applied to the data (see Sect. 2.3). The solid lines indicate isochrones for RGB stars by VandenBerg et al. (2006) with colour transformations by Clem et al. (2004). Their metallicities are indicated in a). The dashed lines indicate dwarf star sequences for different metallicities, $[\mathrm{Fe} / \mathrm{H}]=$ $0.45,-0.05$ and -1 from top to bottom and the dotted line marks the upper envelope for dwarf stars (all lines from Árnadóttir et al., in preparation). The thick line marks the empirically determined limit for the foreground contamination (see Sect. 4.1).

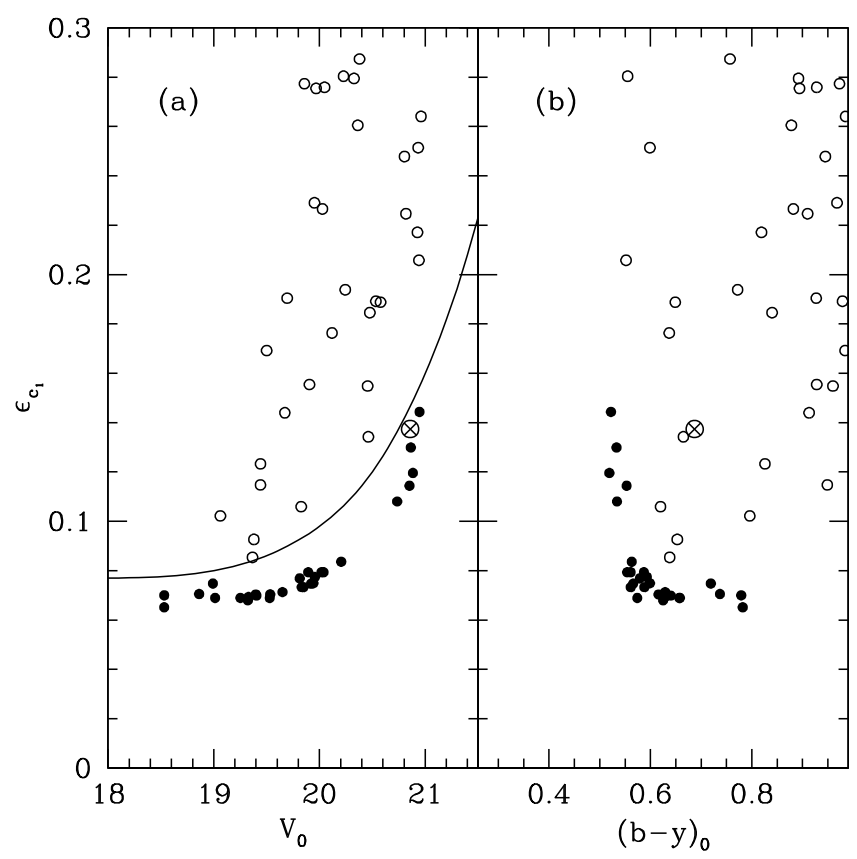

Fig. 13. $\epsilon_{c_{1}}$ vs. $V_{0}$ and $(b-y)_{0}$ for the stars in the RGB region identified in Fig. 12. Symbols are the same as in Fig. 12 and the line in a) is the same as in Fig. 11.

In Fig. 13 we show $\epsilon_{c_{1}}$ vs. $V_{0}$ and $(b-y)_{0}$ for all the stars falling in the RGB region.

In total we find 29 member stars in the RGB region, and 9 in the RHB-AGB region. They are listed in Table 9.
Table 6. Objects with a stellarity index lower than 0.5 , a radial velocity much lower than the mean velocity of the galaxy or $V_{0}>21$.

\begin{tabular}{lcccc}
\hline \hline ID & RA(2000) & $\operatorname{Dec}(2000)$ & $V_{0}$ & $V_{\text {rad }}$ \\
\hline 34489 & 247.66907 & 13.07061 & $20.27 \pm 0.02$ & $\ldots$ \\
$43290^{a}$ & 247.63172 & 12.77969 & $19.79 \pm 0.02$ & $44.9 \pm 3.1$ \\
42668 & 247.69964 & 12.85175 & $19.62 \pm 0.02$ & $-2.8 \pm 1.3$ \\
\hline
\end{tabular}

${ }^{a}$ On top of a galaxy.

Column 1 lists the INT ID. Columns 2 and 3 list the coordinates. Column 4 lists the $V_{0}$ magnitude and its associated error. Column 5 lists the radial velocity and its associated error, both in $\mathrm{km} \mathrm{s}^{-1}$.

Objects with a stellarity index lower than 0.5. As mentioned in Sect. 2.1.5 we found two objects of interest, based on their position in the $c_{1,0}$ vs. $(b-y)_{0}$ diagram, with a stellarity index lower than 0.5. INT 34489 at $V_{0}=20.27$ has sclass $=0.3$ and is located right at the edge of the blue limit. An ocular inspection of the object indicates that it may be a star, but it is slightly elongated on the CCD. INT 43290 at $V_{0}=19.79$ has sclass $=0.35$. In Sect. 3.2 we found that this object lies on top of a galaxy and is therefore contaminated. Given the higher uncertainty of these two objects we do not include them in further analysis. The stars are listed in Table 6.

A possible foreground RGB star. In Fig. 12b we found one star, INT 42668, that fall within the RGB region but it has a radial velocity of $V_{\text {rad }}=-2.8 \mathrm{~km} \mathrm{~s}^{-1}$ (see Sect. 5) and $[\mathrm{Fe} / \mathrm{H}]_{\mathrm{Cal}}=$ -0.5 (see Sect. 8.1). Since it has a radial velocity much lower than the mean velocity of the Hercules dSph galaxy, we identify 

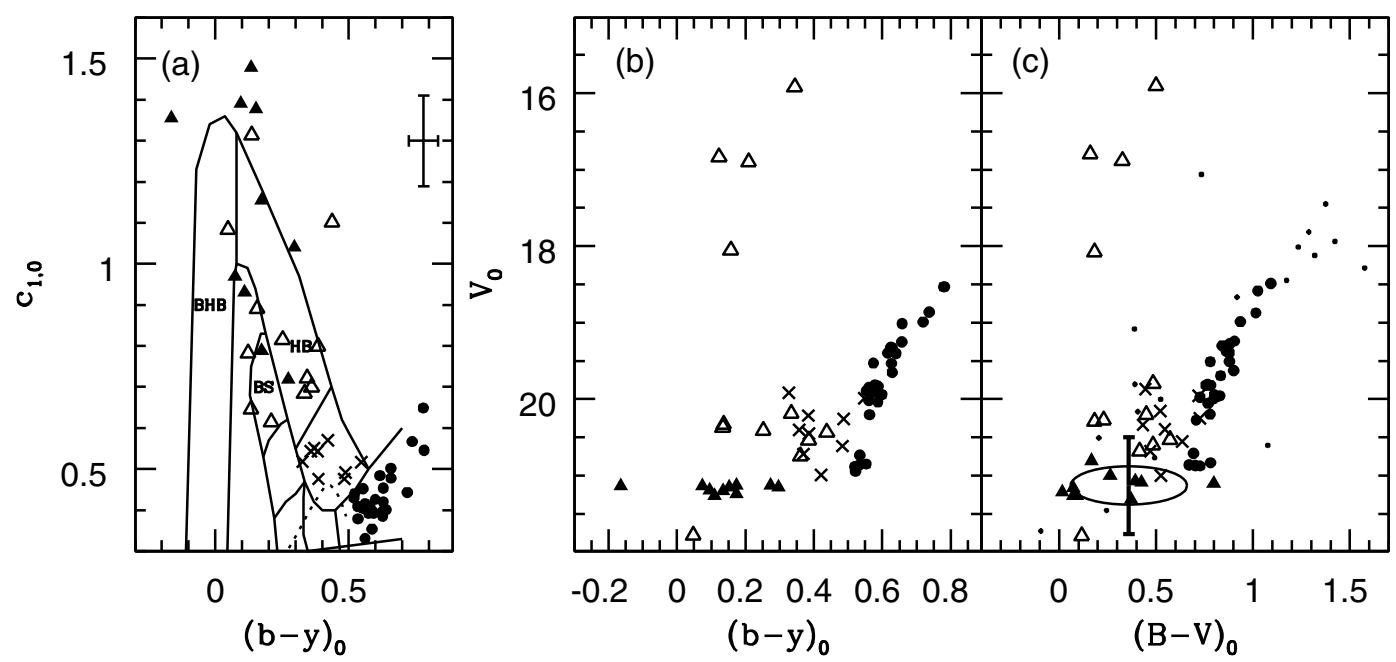

Fig. 14. a) $c_{1,0}$ vs. $(b-y)_{0}$ for the stars in the direction of the Hercules dSph galaxy. BHB: the blue horizontal branch; HB: the remainder of the horizontal branch; BS: the blue stragglers (Schuster et al. 2004). The dotted line marks the upper envelope for dwarf stars. $\bullet$ are stars identified as RGB members and $\times$ are stars identified as RHB-AGB stars based on their evolutionary stage (compare Figs. 9 and 12). $\Delta$ are stars that fall on or are near the HB region but with a large offset to the expected HB magnitude. Filled triangles are stars that fall on or near the HB region and with the expected HB magnitude, they are thus identified as HB member stars. Error-bars in the top right corner are the mean $\epsilon_{c_{1,0}}$ and $\epsilon_{(b-y)_{0}}$ for the stars identified as HB stars. b) Colour-magnitude diagram in the Strömgren system for the stars identified in a). c) Colour-magnitude diagram in the Johson-Cousin UBVRI system for the stars identified in a). The solid ellipse outlines the distribution of variable stars in the Draco dSph galaxy from Bonanos et al. (2004), normalized in $V_{0}$ to fit our HB and · marks variable stars from Bonanos et al. (2004) that fall outside the ellipse. The error bar on the ellipse corresponds to the mean amplitude of the variable stars used to define the ellipse.

this star as a likely foreground RGB star, thus removing it from the sample of member RGB stars. The star is listed in Table 6.

\subsection{Finding the $H B$ and $A G B$ of the Hercules dSph galaxy}

In Fig. 12 we identified RGB members of the Hercules dSph galaxy. Additionally, we found 9 RHB-AGB stars. However, it is possible to use the Strömgren photometry to further explore the RHB-AGB and blue HB stars (BHB). Figure 14a shows the $\mathrm{BHB}, \mathrm{HB}$ and blue straggler (BS) regions in the $c_{1}$ vs. $(b-y)$ plane using the areas defined in Schuster et al. (2004).

In Fig. 14a and $\mathrm{b}$ we show the previously identified RGB and RHB-AGB stars. In addition we show all stars bluewards of $(b-y)_{0}=0.5$ with an $\epsilon_{c_{1}}$ less than the function in Fig. 11 . We further divide these stars into two sets according to their $V$ magnitude. The HB of the Hercules dSph galaxy is roughly at $V_{0}=21.2$ (compare Fig. 6 and Coleman et al. 2007). We therefore define a box with $21<V_{0}<21.4$ and $-0.2<(b-y)_{0}<0.5$ for the potential BHB stars. The second region is given by the magnitude range above and below the first box, i.e. $15<V_{0}<21$ and $21.4<V_{0}<22$ with $-0.2<(b-y)_{0}<0.5$.

Figure $14 \mathrm{a}$ and $\mathrm{b}$ then show these stars both in the $c_{1}$ vs. $(b-y)$ and in the Colour-magnitude diagram. The stars selected in the magnitude range of the $\mathrm{HB}$ are all narrowly spaced in magnitude and they all fall close to the BHB-RHB sequences. Hence we take all 10 of these stars to represent the HB of the Hercules dSph galaxy. We find a mean magnitude for the HB of $V_{0}=21.17 \pm 0.05$ and $V=21.36 \pm 0.05$.

\subsection{A new distance determination to the Hercules dSph galaxy}

We used the relation between the absolute magnitude of the HB and metallicity ( -2.35 dex as derived in Sect. 8$)$, based on globular clusters, as defined in Carretta et al. (2000) to determine the absolute magnitude for the $\mathrm{HB}, M_{\mathrm{HB}}$, in the Hercules dSph galaxy. Using an apparent magnitude for the Hercules HB of $m_{\mathrm{HB}}=21.17 \pm 0.05$ we solve for the distance, $d$

$m_{\mathrm{HB}}-M_{\mathrm{HB}}=-5+5 \cdot \log (d)$.

The errors in the distance determination were calculated using a Monte Carlo simulation. For each parameter with an associated error (apparent HB magnitude, metallicity and constants in the relation between the absolute magnitude and HB and metallicity), a new random apparent HB magnitude, metallicity and constants were calculated, and a new distance based on these parameters was found. This process was repeated 100000 times. In Fig. 15 we show the distribution of the Monte Carlo simulation. As the final errors for the distance, we adopt the lower and the upper sextile of the distribution of simulated distances; the lower sextile cuts off the lowest 16.67 per cent of the data and the upper sextile cuts off the highest 16.67 per cent of the data. We find that Hercules is at a distance of $147_{-7}^{+8} \mathrm{kpc}$ from us.

\subsection{Variable stars in the Hercules dSph galaxy}

In Fig. 14b we see some scatter in the Colour-magnitude diagram above the HB. In agreement with a Besançon model, some of these objects are likely HB stars that belong to the Milky Way. However, the Besançon model can not explain the overabundance of stars seen at $V \sim 20.4$ and $(b-y)_{0} \sim 0.35$. We now investigate if these stars are variable stars that, due to variation in magnitude, position themselves above the HB. Since we do not have more than 3 exposures in the bright filters, $y$ and $b$, it is difficult to determine the variability of these stars as a function of time.

We investigated the variance between the individual exposures in the $y, b, v$ and $u$ filter, but given the small number of exposures we found no strong indication of variability for these stars.

Siegel (2006) report the finding of 15 RR Lyrae variable stars in the Boötes dSph galaxy with periods from $\sim 0.3$ to $\sim 0.9$ days. 


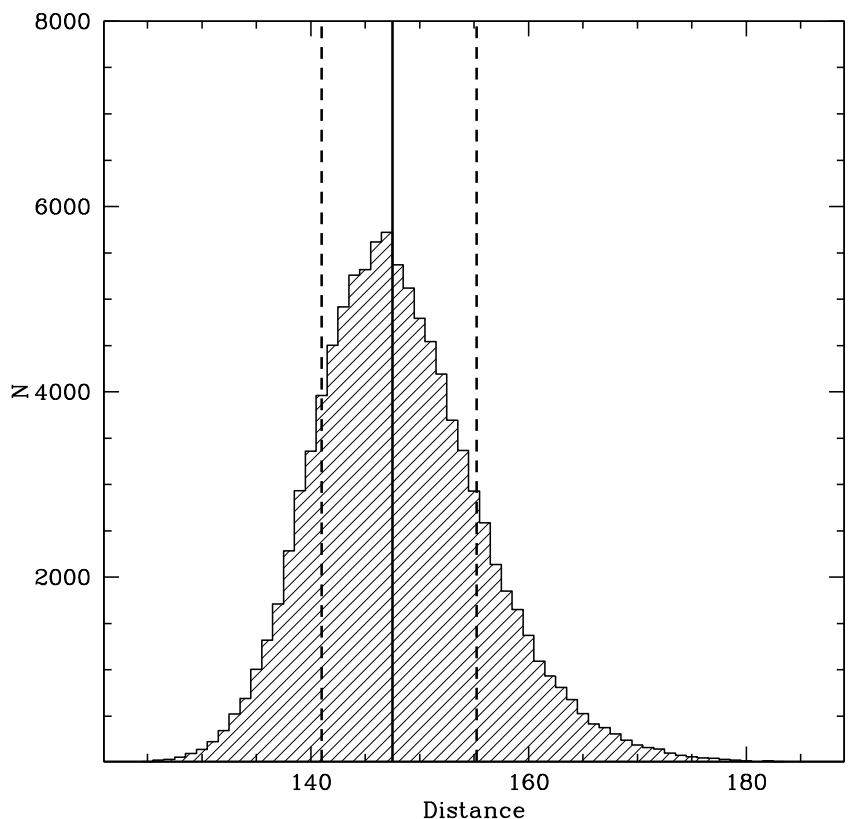

Fig. 15. Determination of the distance to the Hercules dSph galaxy, see Sect. 4.4. Solid line indicates the median distance to the Hercules dSph galaxy. Dashed vertical lines indicate the upper and lower sextile.

Since Boötes, with $M_{V}=-5.8$ (Belokurov et al. 2006), is fainter than the Hercules dSph galaxy, finding a similar number of RR Lyrae stars in our sample is a plausible scenario.

To further investigate the possible presence of variable stars we cross-correlated our photometry with the SDSS photometry in order to obtain ugriz photometry for our stars. We then transformed the ugriz magnitudes on to the Johnson-Cousins $U B V R I$ system using the colour transformations in Jordi et al. (2006). Bonanos et al. (2004) found 163 variable stars in the Draco dSph galaxy. Mean magnitudes in $V, I$ and $B$ and amplitudes in $V$ for these stars are available on-line. We de-reddened the Draco photometry using $E(B-V)=0.027$ (Bonanos et al. 2004) and constructed an ellipse encircling the distribution of the variable stars from Bonanos et al. (2004) in the $V_{0}$ vs. $(B-V)_{0}$ Colourmagnitude diagram (see Fig. 14c).

Using isochrones by Marigo et al. (2008) we find that the HB for two metal-poor, old populations ( -2.3 and $-2.0 \mathrm{dex})$ coincide in $V$. We therefore normalized the positions of the Draco variable stars in $V_{0}$ so that the ellipse is aligned with the mean magnitude of our HB in the Hercules dSph galaxy, Fig. 14c. We also include the variable stars in Draco from Bonanos et al. (2004) that fall outside the ellipse. We see that the positions of the variable stars in the Colour-magnitude diagram for the Draco dSph galaxy are similar to the positions of our open triangles: 1) they have a similar colour range; 2$)$ they are more likely to fall above the HB than below; 3$)$ the spread in $V_{0}$ at $(B-V)_{0} \sim 0.5$ is similar for both data sets.

Our conclusion from this analysis is that the 8 stars, fainter than $V_{0}=19$, likely are variable stars in the Hercules dSph galaxy. These stars are listed in Table 7.

\section{Determining the systemic velocity of the Hercules dSph galaxy}

Using radial velocity measurements to separate the stars belonging to a dSph galaxy from foreground stars has proven to be an efficient method for membership determination. However, for
Table 7. Possible variable stars in the Hercules dSph galaxy identified in Sect. 4.5.

\begin{tabular}{ccccc}
\hline \hline ID & RA(2000) & Dec(2000) & $V_{0}$ & $(b-y)_{0}$ \\
\hline 11718 & 247.84445 & 12.60114 & $20.44 \pm 0.02$ & $0.44 \pm 0.04$ \\
22960 & 247.45022 & 12.59089 & $20.19 \pm 0.02$ & $0.33 \pm 0.04$ \\
33388 & 247.74828 & 12.96788 & $20.38 \pm 0.04$ & $0.13 \pm 0.06$ \\
41701 & 247.78819 & 12.78892 & $20.74 \pm 0.03$ & $0.36 \pm 0.04$ \\
41807 & 247.77753 & 12.76208 & $20.34 \pm 0.02$ & $0.14 \pm 0.04$ \\
42113 & 247.74934 & 12.76745 & $20.41 \pm 0.02$ & $0.25 \pm 0.04$ \\
42503 & 247.71592 & 12.77974 & $21.78 \pm 0.04$ & $0.05 \pm 0.06$ \\
43193 & 247.64148 & 12.79060 & $20.54 \pm 0.03$ & $0.39 \pm 0.04$ \\
\hline
\end{tabular}

Column 1 lists the INT ID. Columns 2 and 3 list their coordinates. Columns 4 and 5 list the Strömgren magnitude $V_{0}$ and colour $(b-y)_{0}$ and their associated errors, respectively.

the Hercules dSph galaxy this method is complicated since this galaxy has a systemic velocity that falls within the velocity distribution of the foreground dwarf stars in the Milky Way.

In Fig. 8 we show the velocity distribution for our observations together with a Besançon model in the direction of the Hercules dSph galaxy. As can be seen, the velocity peak of the Hercules dSph galaxy lies within the velocity distribution of the Milky Way galaxy. A sample of member stars, identified as members based only on radial velocity will therefore contain a non-negligible number of foreground stars. However, adding knowledge about the evolutionary stage of the stars means that we can eliminate the foreground dwarf stars (compare Sect. 4.2) and obtain a clean sample which can be used to determine the systemic velocity and velocity dispersion for the dSph galaxy.

In order to illustrate the importance of knowing the evolutionary stage of the star we will first consider only the radial velocities as a means to define a sample of stars belonging to the dSph galaxy. After that we will add knowledge about the evolutionary stage to clean the sample further.

\subsection{Using only radial velocities to select $R G B$ members}

We used the maximum likelihood method described in Walker et al. (2006) to determine the mean heliocentric velocity and internal velocity dispersion for stars in the direction of the Hercules dSph galaxy.

The natural logarithm of the probability function defined in Walker et al. (2006) was maximized

$\ln (p)=-\frac{1}{2} \sum_{i=1}^{N} \ln \left(\sigma_{i}^{2}+\sigma_{p}^{2}\right)-\frac{1}{2} \sum_{i=1}^{N} \frac{\left(v_{i}-u\right)^{2}}{\left(\sigma_{i}^{2}+\sigma_{p}^{2}\right)}-\frac{N}{2} \ln (2 \pi)$.

In each iteration we rejected stars deviating by more than $3 \sigma$ as they are likely not members. As can be seen in Fig. 8, the objects targeted with FLAMES span a broad range of velocities. For the first pass through the maximum likelihood iteration we selected stars with $10 \mathrm{~km} \mathrm{~s}^{-1}<V_{\text {rad }}<70 \mathrm{~km} \mathrm{~s}^{-1}$. The maximization converged after 1 iteration. This method gives us a mean velocity of $40.87 \pm 1.42 \mathrm{~km} \mathrm{~s}^{-1}$ with a dispersion of $7.33 \pm 1.08 \mathrm{~km} \mathrm{~s}^{-1}$. Stars deviating by less than $3 \sigma$ from the velocity could be considered as possible Hercules dSph galaxy members. We find 32 stars in this velocity range.

\subsection{Weeding out foreground dwarf stars with the same velocity as the Hercules dSph galaxy}

One of the 32 stars found in Sect. 5.1 (excluding the three stars with sclass < 0.5), INT 42568, lies on the edge of the WFC 


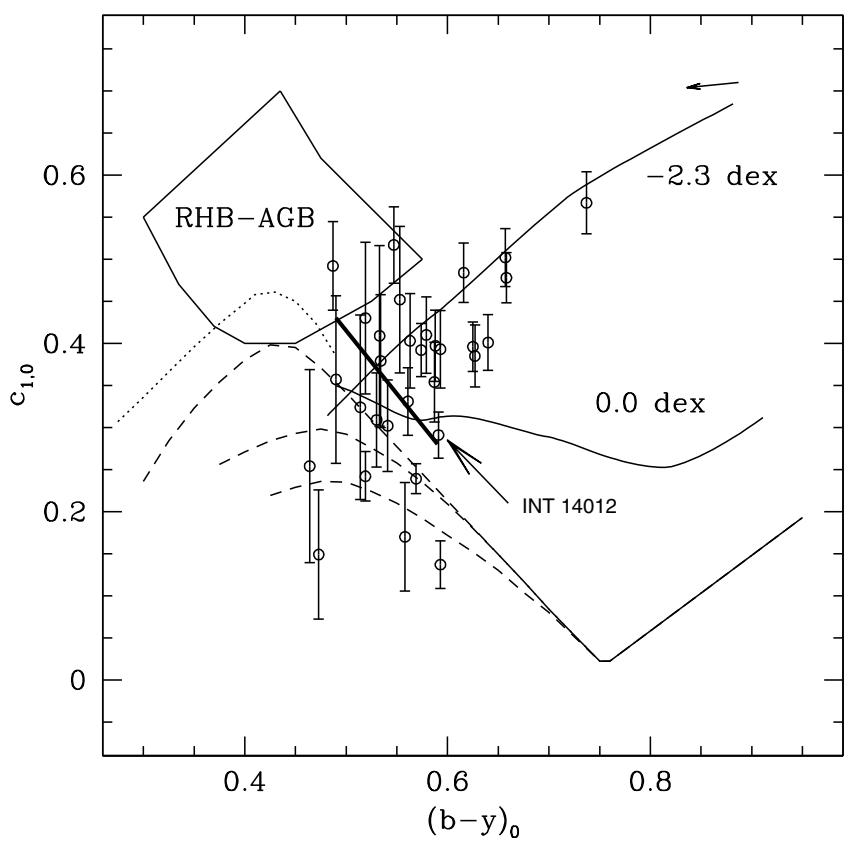

Fig. 16. $c_{1,0}$ vs. $(b-y)_{0}$ diagram for stars considered as members from the radial velocity measurement alone (see Sect. 5.1 ). The thin solid lines indicate the isochrones for RGB stars by VandenBerg et al. (2006) with colour transformations by Clem et al. (2004). The thin dashed lines indicate dwarf star sequences for different metallicities, $[\mathrm{Fe} / \mathrm{H}]=0.45$, -0.05 and -1 top to bottom and the dotted line marks the upper envelope for dwarf stars (all lines from Árnadóttir et al., in preparation). The thick solid line is our lower limit for identification of RGB stars (see Sect. 4). The arrow in the top right corner indicates the magnitude and direction of the de-reddening applied to the data (see Sect. 2.3).

CCD \#4 and does not have any photometry available and is hence excluded from the following discussions. In Fig. 16 we plot $c_{1,0}$ vs. $(b-y)_{0}$ for the 31 stars considered as possible members based on the radial velocities. We find the following

- out of the 31 stars 10 fall on or below the dwarf sequences and are therefore excluded;

- of the remaining 21 stars, 18 are RGB members, 2 fall in the RHB-AGB region;

- the last star falls below the RGB solar isochrone but is redder than the blue limit (compare Figs. 12 and 16). This star is marked in Fig. 16 (INT 14012). We consider this star as a member (but see discussion below).

Hence, we find about 30 per cent contamination by foreground stars in our sample. For the 19 RGB stars with the right evolutionary stage, we re-derive the mean velocity and dispersion using the maxiumum likelihood method. We note that star INT 14012 was rejected during the iteration due to the $3 \sigma$ limit. This star is not included in the final sample of RGB stars. We note that one of the remaining 18 stars, INT 42170, has a velocity just outside the re-derived more narrow $3 \sigma$ limit. Since it falls short of this limit by only $1.32 \mathrm{~km} \mathrm{~s}^{-1}$ and the error in the velocity for the star is $4.77 \mathrm{~km} \mathrm{~s}^{-1}$, we keep it in the final sample. This star could also be a binary, which would explain its deviating velocity. We found no stars that are likely members based on the Strömgren photometry, but non-members based on the radial velocity measurement.

We find a mean final systemic velocity of $45.20 \pm 1.09 \mathrm{~km} \mathrm{~s}^{-1}$ with a dispersion of $3.72 \pm 0.91 \mathrm{~km} \mathrm{~s}^{-1}$. Our conclusion is that all stars, except INT 14012, with the right evolutionary stage fall within $3 \sigma$ of the systemic velocity.

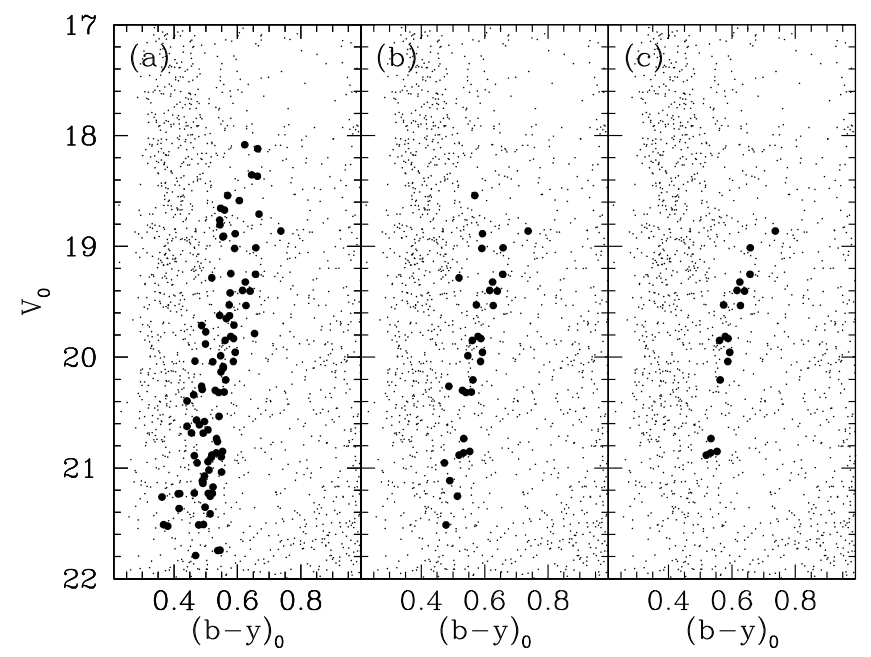

Fig. 17. Colour-magnitude diagrams. a) Objects targeted with FLAMES are shown as large •. Small dots indicate stars from Fig. 6a. b) Stars that have radial velocities within $3 \sigma$ of the systemic velocity. c) Stars that are members based on the evolutionary stage and have measured velocities within $3 \sigma$ of the systemic velocity (see Sect. 5.2).

Figures $17 \mathrm{a}$ to $\mathrm{c}$ show the Colour-magnitude diagrams for objects targeted with FLAMES, stars identified as members from the radial velocity measurement only and, finally, stars identified as members from the radial velocity measurement and using photometry to weed out foreground dwarf stars.

\section{Final sample}

Our final sample of RGB, AGB and HB Hercules member stars was defined as follows: 1) first we select the RGB stars with the right evolutionary stage and a radial velocity within $\sim 3 \sigma$ of the systemic velocity (compare Sect. 5.2 and Fig. 17c); 2) to these stars we add stars without spectroscopic measurements, which were selected as RGB, AGB, or HB stars as determined from photometry (compare Sect. 4.2). These stars are listed in Table 9. Figures $18 \mathrm{a}$ and $\mathrm{b}$ show the Colour-magnitude diagrams for the final sample.

\section{A comparison with previous velocity determinations}

Simon \& Geha (2007) have obtained radial velocities for 86 stars in the direction of the Hercules dSph galaxy. In order to avoid including foreground dwarf stars they used measurements of the strength of the Na I lines at 818.3 and $819.5 \mathrm{~nm}$ to distinguish between dwarf and giant stars. As discussed in Schiavon et al. (1997) the strength of these lines depend on the gravity of the star. Hence, it enables a distinction between dwarf and giant stars. Out of the 86 stars 29 were identified as members based on the measurements of the strength of the $\mathrm{Na} I$ lines.

There are 21 stars in common, including field dwarf stars, between our spectroscopic study and Simon \& Geha $(2007)^{2}$. In Fig. 19 we show the difference, in units of Gaussian $\sigma$, in stellar velocity for these stars (see Kleyna et al. 2002, for a similar study of the Draco dSph galaxy). We note that the velocity measurements are in agreement, and that there are no obvious outliers, hence there is no evidence for binaries in this sample.

\footnotetext{
2 J. Simon and M. Geha have kindly provided the relevant data so that we could do this analysis.
} 


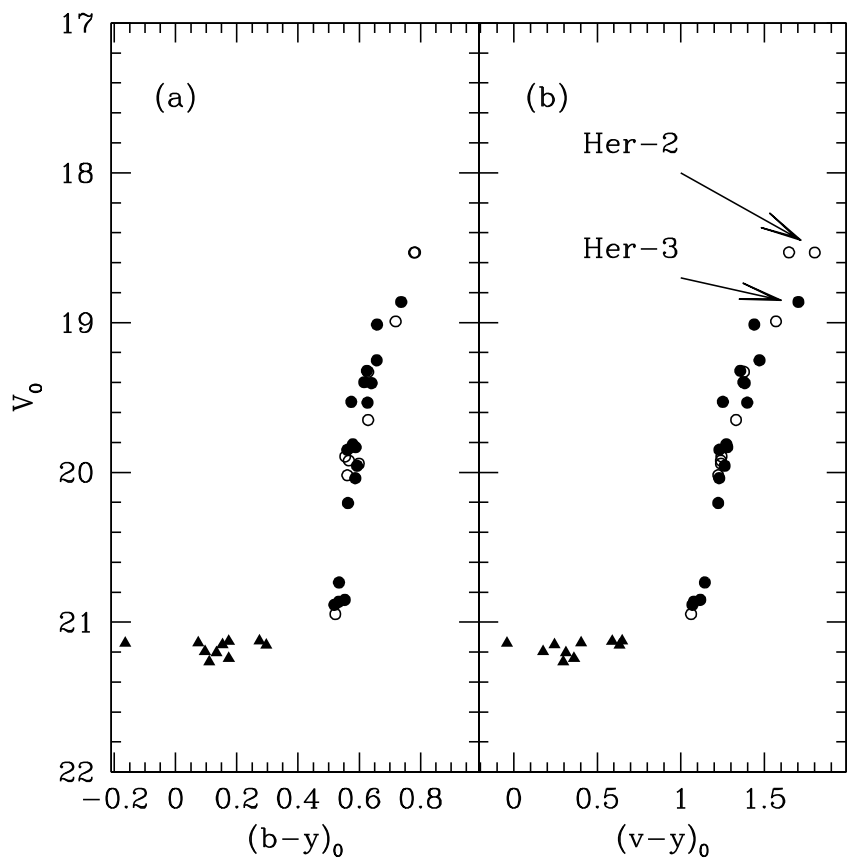

Fig. 18. Final Colour-magnitude diagrams for the Hercules dSph galaxy. a) $V_{0}$ vs. $(b-y)_{0}$. b) $V_{0}$ vs. $(v-y)_{0}$. $\bullet$ are members based on the evolutionary stage and have measured velocities within $3 \sigma$ of the systemic velocity (see Sect. 5.2). ○ are stars that do not have radial velocity measurements but are members according to their evolutionary stage (see Sect. 4.2). The filled triangles are stars identified as HB stars (see Sect. 4.3). Her-2 and Her-3 are discussed in Sect. 8.3. Note that there are two stars in a) at $V_{0} \sim 18.5$ that split in b).

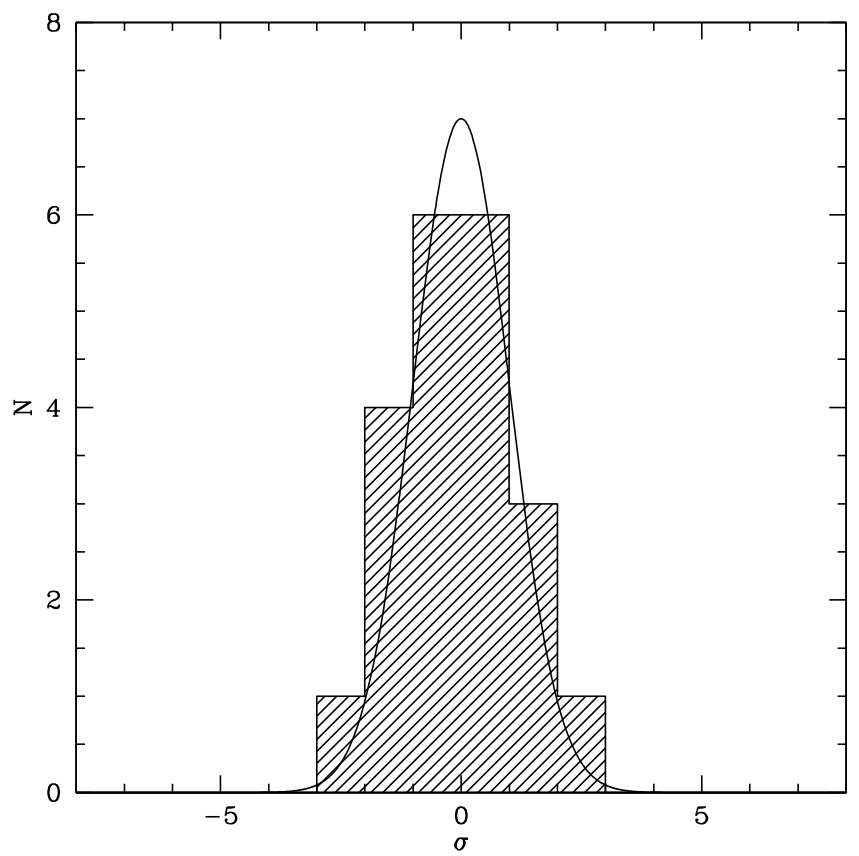

Fig. 19. Difference, in units of Gaussian $\sigma$ (see Sect. 7), between our measured stellar velocities and the velocities from Simon \& Geha (2007). Note that this histogram includes both members and nonmembers. The solid line indicates a Gaussian with $\sigma=1$.

However, given the limited time sampling of the combined spectroscopy, and the fact that there are stars in our spectroscopic study for which there are no velocity measurement in Simon \& Geha (2007), we cannot rule out the presence of binaries in our

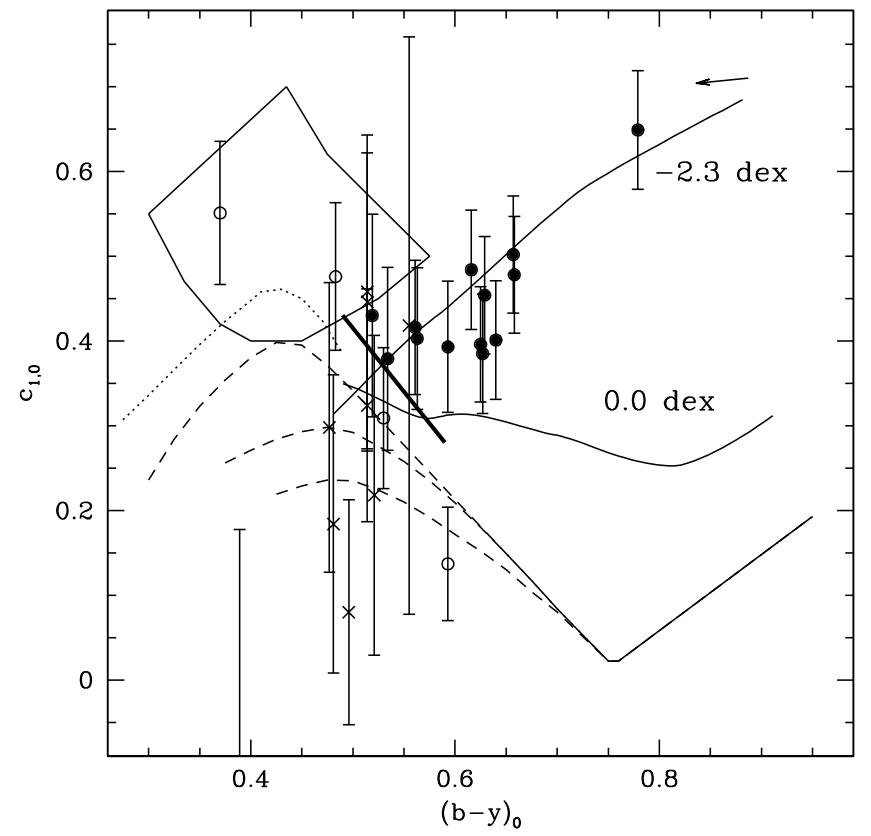

Fig. 20. Strömgren $c_{1,0}$ vs. $(b-y)_{0}$ diagram for the stars from Simon \& Geha (2007). • are stars considered as RGB members and $\circ$ are stars identified as non-RGB members based on our Strömgren photometry (see Sect. 4.2). $\times$ are stars that have $V_{0}>21$, hence we have not considered their evolutionary stage as they are too faint. The thin solid lines indicates the isochrones for RGB stars by VandenBerg et al. (2006) with colour transformations by Clem et al. (2004). The thin dashed lines indicates dwarf star sequences for different metallicities, $[\mathrm{Fe} / \mathrm{H}]=0.45$, -0.05 and -1 top to bottom and the dotted line marks the upper envelope for dwarf stars (all lines from Árnadóttir et al., in preparation). The thick solid line is our lower limit for identification of RGB stars. The arrow in the top right corner indicates the magnitude and direction of the de-reddening applied to the data (see Sect. 2.3).

study. The presence of binaries could inflate the observed velocity dispersion by a small amount (e.g. Olszewski et al. 1996).

For the stars in common between our photometric study and Simon \& Geha (2007) we show a $c_{1}$ vs. $(b-y)$ diagram in Fig. 20. From this comparison we find the following

- out of the 29 stars considered as members in Simon \& Geha (2007), 2 fall in the RHB-AGB region and are therefore excluded as RGB members;

- of the remaining 27 stars, 12 have $V_{0}>21$, hence we have not considered their evolutionary stage as they are too faint. 4 out of the 12 stars with $V_{0}>21$ fall outside the limits of Fig. 20;

- 2 of the remaining stars fall on or below the dwarf sequences and are therefor excluded as members of the Hercules dSph galaxy;

- thus 13 stars remain that are considered as RGB members based on Strömgren photometry.

For the 13 RGB members in common between us and Simon \& Geha (2007), using the velocities from Simon \& Geha (2007), we find a mean systemic velocity of $46.10 \pm 1.30 \mathrm{~km} \mathrm{~s}^{-1}$ with a dispersion of $4.01 \pm 1.08 \mathrm{~km} \mathrm{~s}^{-1}$.

For 10 of the 13 RGB members we have determined radial velocities (see Sect. 2.2.2). Given the small offset in velocity determination between our study and Simon \& Geha (2007), see Fig. 19, it is possible to include the velocities of the 3 stars for which we have not obtained a velocity measurement into our calculation of the mean velocity. We find a mean systemic velocity 


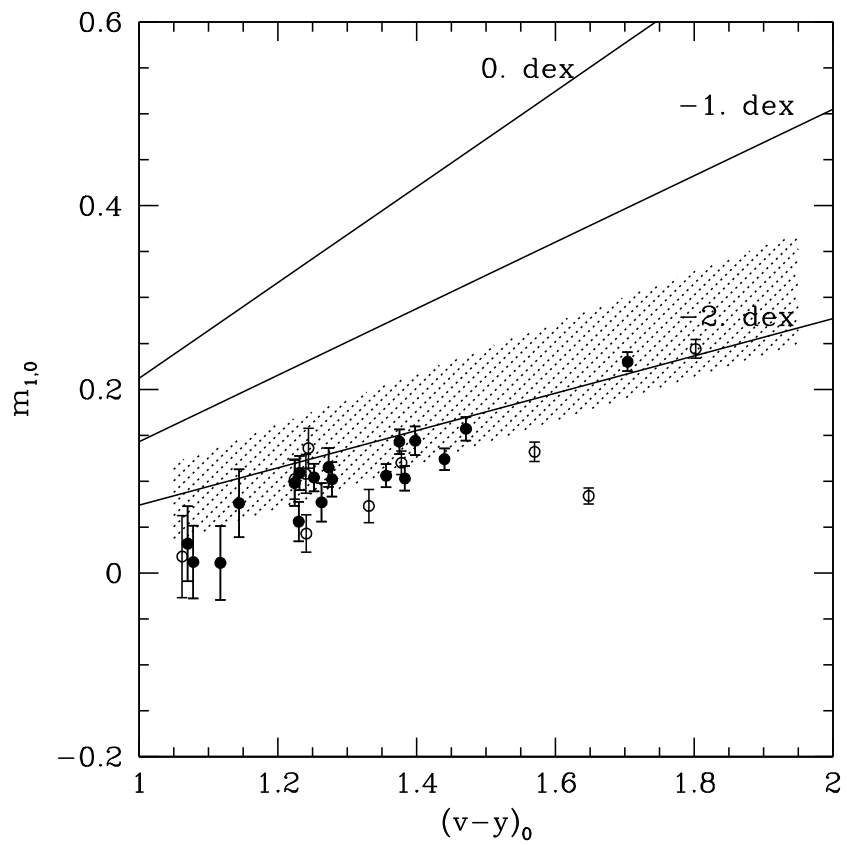

Fig. 21. $m_{1,0}$ vs. $(v-y)_{0}$ for the Hercules dSph galaxy. • are members based on the evolutionary stage as derived from photometry and they have radial velocities within $3 \sigma$ of the systemic velocity for the Hercules dSph galaxy (see Sect. 5.2). The $\circ$ are stars that do not have radial velocity measurements but are members according to our photometric criteria as developed in Sect. 4.2. The solid lines are isometallicity lines based on Eq. (14) with metallicities as indicated. The shaded area indicates the distribution of RGB stars from Faria et al. (2007) for the Draco dSph galaxy, adopted from their Fig. 19.

of $44.95 \pm 1.02 \mathrm{~km} \mathrm{~s}^{-1}$ with a dispersion of $3.84 \pm 0.85 \mathrm{~km} \mathrm{~s}^{-1}$ which is in agreement with our result from Sect. 5.2.

There are 15 stars in our final sample of RGB members (based on the evolutionary stage) that do not have radial velocities measured in Simon \& Geha (2007).

Measuring the strength of the $\mathrm{Na}$ I to exclude foreground dwarf stars is valid for $(V-I)>1$ (Gilbert et al. 2006; Koch et al. 2008c). This corresponds to $(b-y)>0.55$ and is confirmed by our comparison where the 16 stars that are bluer than this limit are identified as either foreground dwarf stars or HB stars by the Strömgren $c_{1}$ index.

\section{Metallicities}

\subsection{Metallicities based on Strömgren photometry}

\subsubsection{Determination of metallicities}

The Strömgren filters have proven useful to estimate stellar metallicities for RGB stars via the $m_{1}$ index (e.g. Richtler 1989),

$m_{1}=(v-b)-(b-y)$.

A review of the Strömgren metallicity calibrations for RGB stars, available at the time, is provided by Faria et al. (2007). They adopt the Hilker (2000) calibration for their RGB stars in the Draco dSph galaxy. Since the Hilker (2000) calibration is not valid for $[\mathrm{Fe} / \mathrm{H}]<-2.0 \mathrm{dex}$ and the newly found ultrafaint $\mathrm{dSph}$ galaxies such as Hercules are metal-poor, we adopt the semi-empirical calibration by Calamida et al. (2007) onto the metallicity scale of Zinn \& West (1984) as this calibration is valid at least down to -2.4 dex.

Figure 21 shows the $m_{1,0}$ vs. $(v-y)_{0}$ plane for the stars identified as RGB stars in the Hercules dSph galaxy.
Equation (14), adopted from Calamida et al. (2007), is used to convert $m_{1,0}$ and $(v-y)_{0}$ to $[\mathrm{Fe} / \mathrm{H}]_{\mathrm{Cal}}$ for the RGB stars.

$[\mathrm{Fe} / \mathrm{H}]_{\mathrm{Cal}}=\frac{\left(m_{1}+b_{1}(v-y)+b_{2}\right)}{\left(b_{3}(v-y)+b_{4}\right)}$

where $b_{1}=-0.521 \pm 0.001, b_{2}=0.309, b_{3}=0.159 \pm 0.001$ and $b_{4}=-0.09 \pm 0.002$ (note that $b_{2}$ does not have an error estimate in Calamida et al. 2007).

For comparison we re-calculated the stellar metallicities for the Draco RGB sample from Faria et al. (2007) using the calibration by Calamida et al. (2007, see Fig. 21). We found that the Draco dSph galaxy has a mean metallicity of -2.0 dex instead of -1.74 dex as calculated by Faria et al. (2007). A comparison between the metallicities derived using the older Hilker (2000) calibration and $[\mathrm{Fe} / \mathrm{H}]_{\mathrm{Cal}}$ shows an offset of $\sim 0.3 \mathrm{dex}$, where $[\mathrm{Fe} / \mathrm{H}]_{\mathrm{Cal}}$ is more metal-poor.

In Sect. 2.3 we described how we correct the photometric magnitudes for interstellar extinction using $E(B-V)=0.062$. As a test we re-calculated $[\mathrm{Fe} / \mathrm{H}]_{\mathrm{Cal}}$ using $E(B-V)=0.032$ and $E(B-V)=0.092$, which correspond to an uncertainty of $\pm 0.03 \mathrm{mag}$. We found that $E(B-V)=0.032$ increased and $E(B-$ $V)=0.092$ decreased the metallicity by $\sim 0.1$ dex and $\sim 0.12$ dex, respectively.

\subsubsection{Error estimates}

Following Faria et al. (2007), the errors in $[\mathrm{Fe} / \mathrm{H}]_{\mathrm{Cal}}$ were calculated using a Monte Carlo simulation.

The simplest version of this approach would be to calculate the errors in metallicities taking into account only the measurement errors from PHOT (merr) and propagate those errors through Eq. (14) (this is the approach used in Faria et al. 2007). A more involved approach would be to include also the uncertainties in zeropoints, extinction coefficients and colour terms. For comparison we have calculated the errors using both approaches.

In both cases we used the following approach to calculate the errors. For every star identified as an RGB member of the Hercules dSph galaxy, new random magnitudes ( $v, b$ and $y$ ) were calculated from Gaussian probability distributions with standard deviations equal to that of the photometric error for each filter using a Box-Muller transformation. $m_{1,0}$ and $(v-y)_{0}$ were recalculated from these new magnitudes and used to derive a new metallicity for the star. This process was then repeated $10^{5}$ times for each star and since the distributions of the new metallicities are not Gaussian around the original $[\mathrm{Fe} / \mathrm{H}]$, a standard deviation based on the sextiles (which is equivalent to $1 \sigma$ in the case of a Gaussian distribution) was calculated for each star from the distribution of simulated $[\mathrm{Fe} / \mathrm{H}]$. This sextile standard deviation is our error in $[\mathrm{Fe} / \mathrm{H}]_{\mathrm{Cal}}$ (compare Faria et al. 2007).

In Fig. 22a we show the errors in $[\mathrm{Fe} / \mathrm{H}]_{\mathrm{Cal}}$ as a function of $V_{0}$. Note that the five faintest stars have significantly larger errors. Also note that the errors are much larger when the uncertainties in zeropoints, extinction coefficients and colour terms are taken into account. We find that it is the errors in the $b$ filter that is the largest contributor to the error in $[\mathrm{Fe} / \mathrm{H}]_{\mathrm{Cal}}$ when the uncertainties in zeropoints, extinction coefficients and colour terms are taken into account (compare Table 3). The errors in the $b$ filter account for almost 50 per cent of the total error. 

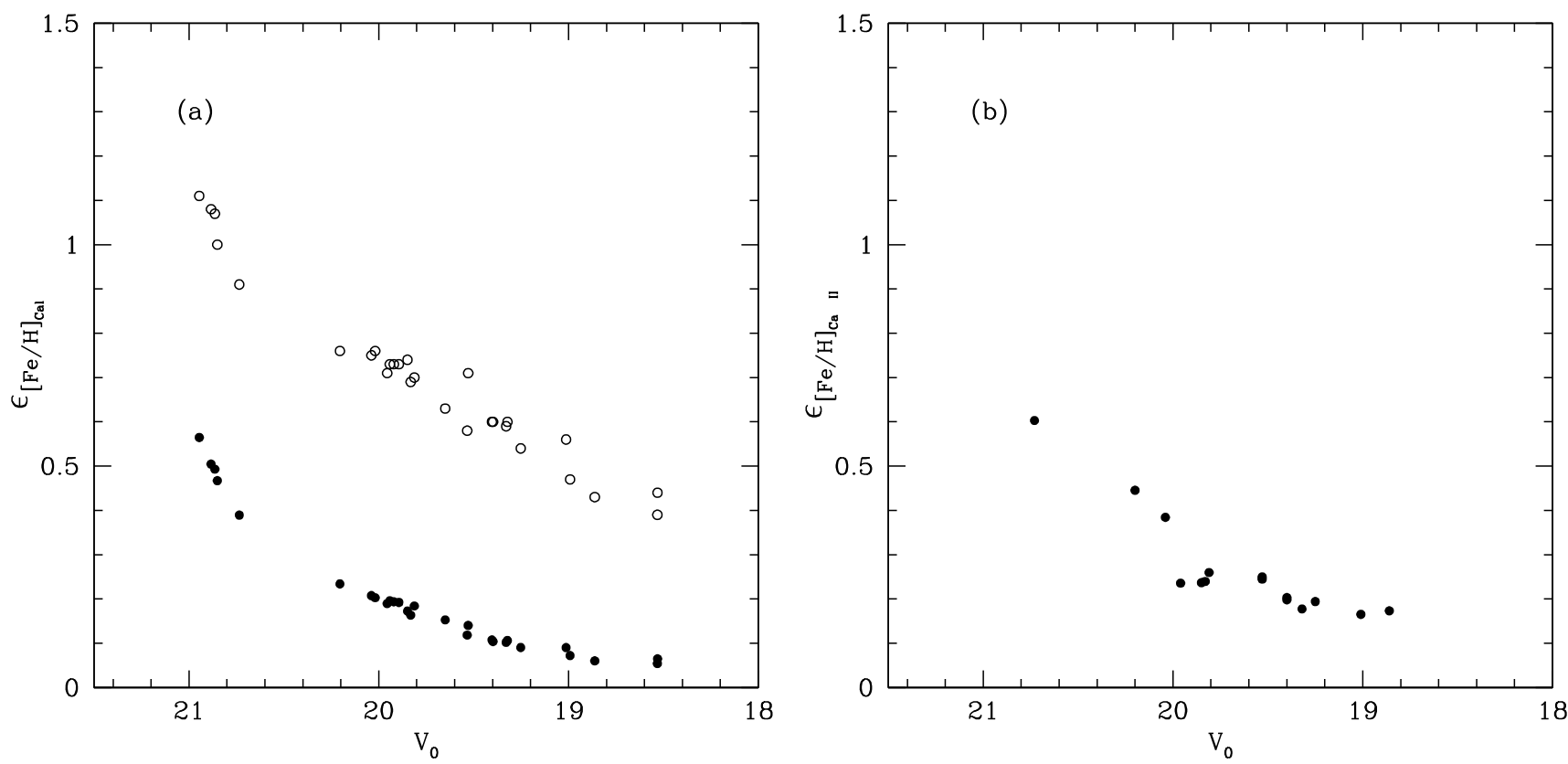

Fig. 22. a) Errors in the $[\mathrm{Fe} / \mathrm{H}]_{\mathrm{Cal}}$ for all stars identified as RGB members of the Hercules dSph galaxy in Sect. 8.1.2. • indicates the errors if only merr and the uncertainties in the coefficients of Eq. (14) are included. $\circ$ indicates the errors if merr, the uncertainties in the coefficients of Eq. (14), and the uncertainties in zeropoints, extinction coefficients and colour terms are included. b) Errors in the $[\mathrm{Fe} / \mathrm{H}]_{\text {CaII }}$ as derived in Sect. 8.2.2 for stars identified as RGB members.

\subsection{Metallicities based on Ca II IR triplet lines}

\subsubsection{Determination of metallicities}

For those RGB stars for which we have FLAMES observations we also determined metallicities $\left([\mathrm{Fe} / \mathrm{H}]_{\mathrm{Ca} \text { II }}\right)$ from measurements of the equivalent width of the Ca II IR triplet lines at $\lambda=849.8,854.2$ and $866.2 \mathrm{~nm}$. We follow Rutledge et al. (1997b) in defining the line strength of the Ca II IR triplet lines as the weighted sum of the $W$, with lower weights for the weaker lines

$\sum W=0.5 \cdot W_{1}+W_{2}+0.6 \cdot W_{3}$

where $W_{1}, W_{2}$ and $W_{3}$ are the widths of the individual Ca II IR triplet lines in the order of increasing wavelength.

As discussed e.g. in Rutledge et al. (1997a), the strength of the Ca II IR triplet lines depend not only on metallicity but also on the surface gravity and effective temperature of the star. It is possible to remove the effect of gravity and temperature to first order by taking into account the position of the star on the RGB. This is done by defining the reduced $W$ as

$W^{\prime}=\sum W+0.64( \pm 0.02)\left(V-V_{\mathrm{HB}}\right)$

where $\left(V-V_{\mathrm{HB}}\right)$ is the difference between the $V$ magnitude of the star and the $V$ magnitude of the horizontal branch $\left(V_{\mathrm{HB}}\right)$. The final $[\mathrm{Fe} / \mathrm{H}]_{\mathrm{Ca} \text { II }}$ were calculated using the calibration by Rutledge et al. (1997a) onto the metallicity scale of Carretta \& Gratton (1997). The Rutledge et al. (1997a) calibration reads as follows

$[\mathrm{Fe} / \mathrm{H}]_{\mathrm{Ca} I \mathrm{I}}=-2.66( \pm 0.08)+0.42( \pm 0.02) W^{\prime}$.

Of the 18 RGB stars considered as members based on the evolutionary stage and which have measured velocities within $3 \sigma$ of the systemic velocity, 3 have too low $S / N$ for measurements of the $W$ and are therefore excluded from this metallicity determination.

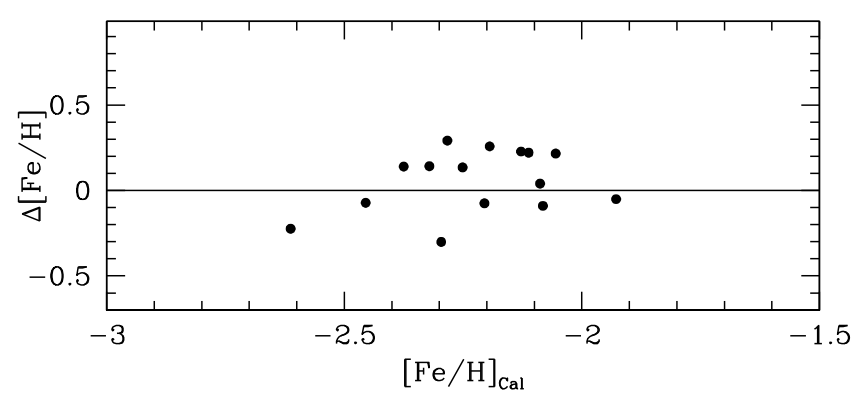

Fig. 23. A comparison between metallicities derived from the $m_{1}$ index and those derived from Ca II IR triplet line measurements. $\Delta[\mathrm{Fe} / \mathrm{H}]=$ $[\mathrm{Fe} / \mathrm{H}]_{\mathrm{Cal}}-[\mathrm{Fe} / \mathrm{H}]_{\mathrm{CaII}}$. The offset is $0.06 \mathrm{dex}$ with a scatter of $0.18 \mathrm{dex}$.

Figure 23 shows a comparison between our two metallicity estimates. We find that $[\mathrm{Fe} / \mathrm{H}]_{\mathrm{Cal}}$ is on average 0.06 dex larger than $[\mathrm{Fe} / \mathrm{H}]_{\mathrm{CaII}}$ with a scatter of $0.18 \mathrm{dex}$. This scatter is consistent with the typical measurement uncertainty of $[\mathrm{Fe} / \mathrm{H}]_{\mathrm{CaII}}$ (see below). In conclusion, the agreement between the photometric and spectroscopic metallicities is very good. Since the calibration by Calamida et al. (2007) is valid to at least $[\mathrm{Fe} / \mathrm{H}]=$ -2.4 , we conclude that the extrapolation for the $[\mathrm{Fe} / \mathrm{H}]_{\mathrm{Ca} \text { II }}$ to $[\mathrm{Fe} / \mathrm{H}] \sim-2.4$ is valid (compare Battaglia et al. 2008). We note that the abundance scale of Zinn \& West (1984), as used by Calamida et al. (2007), in general has $\sim 0.2$ dex lower metallicities at around -2.0 dex than the abundance scale of Carretta \& Gratton (1997) as used by Rutledge et al. (1997a).

\subsubsection{Error estimates}

Following Battaglia et al. (2008), we define the error due to random noise in the measurement of the $W$ as

$\Delta W=\frac{\sqrt{1.5 \cdot F W H M}}{S / N}$ 
where $F W H M$ is the Gaussian full-width-half maximum. The $S / N$ varies from $\sim 24$ for the brightest star to $\sim 5$ for the faintest star. The errors in $[\mathrm{Fe} / \mathrm{H}]_{\text {Ca II }}$ were calculated following a Monte Carlo simulation procedure similar to the one used in Sect. 8.1.2. The process was repeated 100000 times. As the final error for $[\mathrm{Fe} / \mathrm{H}]_{\mathrm{CaII}}$, we adopt the standard deviation calculated from the distribution of simulated $[\mathrm{Fe} / \mathrm{H}]_{\mathrm{CaII}}$. In Fig. $22 \mathrm{~b}$ we show the errors in $[\mathrm{Fe} / \mathrm{H}]_{\mathrm{CaII}}$ as a function of $V_{0}$.

\subsection{A comparison with metallicities determined in other studies}

Koch et al. (2008b) obtained high resolution spectroscopy of two stars in the Hercules dSph galaxy, Her-2 and Her-3. These stars correspond to our stars INT $42241\left(V_{0}=18.53\right)$ and INT $41082\left(V_{0}=18.86\right)$. The stars are marked in Fig. 18 . Koch et al. (2008b) find $[\mathrm{Fe} / \mathrm{H}]=-2.02$ and -2.04 for Her-2 and Her-3, respectively.

In Sect. 4.2 we identify both of these stars as RGB stars and members of the Hercules dSph galaxy. We only have a radial velocity for one of the stars, INT 41082 (Her-3). The velocity of this star falls within $3 \sigma$ of our final systemic velocity (see Sect. 5.2). For this star we find $[\mathrm{Fe} / \mathrm{H}]_{\mathrm{Cal}}=-1.93$ and $[\mathrm{Fe} / \mathrm{H}]_{\mathrm{CaII}}=-1.88$. For INT $42241($ Her-2) we derive $[\mathrm{Fe} / \mathrm{H}]_{\mathrm{Cal}}=-1.96$.

Hence, there is a difference of 0.11 and 0.06 dex, respectively, when high resolution spectroscopy $\mathrm{Fe}$ abundances and metallicities derived from Strömgren photometry are compared. This must be regarded as excellent agreement given the complexities in analysing spectra of such cool, evolved giant stars and the general simplifications made when using calibrations of photometric measurements to obtain estimates of stellar metallicities. It should also be noted that an overestimate in the reddening of a few hundredths would easily account for this difference (compare Sect. 8.1).

Kirby et al. (2008b) studied 20 stars in the direction of the Hercules dSph galaxy. Their metallicities are based on a recently developed automated spectrum synthesis method that takes the information in the whole spectrum into account (Kirby et al. 2008a). The method was originally developed for globular clusters in the Milky Way and was then applied to ultra-faint dSph galaxies in Kirby et al. (2008b). [Fe/H] from Kirby et al. (2008b) will henceforth be referred to as $[\mathrm{Fe} / \mathrm{H}]_{\text {Kirby }}$. We have crosscorrelated our photometry with their 20 stars and found the following (see Fig. 24)

- out of the 20 stars considered as members by Kirby et al. (2008b), 2 fall in the RHB-AGB region and are therefore excluded as RGB members;

- of the remaining 18 stars, 4 have $V_{0}>21$, hence we have not considered their evolutionary stage but all indications are that at least three of them are foreground dwarf stars, compare Fig. 24;

- 2 of the 14 remaining stars fall on or below the dwarf sequences and are therefore foreground dwarf stars.

For the remaining 12 stars we find a mean metallicity of $[\mathrm{Fe} / \mathrm{H}]_{\mathrm{Cal}}=-2.25 \pm 0.20 \mathrm{dex}$ (Sect. 8.1) and a mean metallicity based on the values from Kirby et al. (2008b) $[\mathrm{Fe} / \mathrm{H}]_{\mathrm{Kir}}=$ $-2.70 \pm 0.47$ dex. Figure 25 shows the difference between $[\mathrm{Fe} / \mathrm{H}]_{\mathrm{Cal}}$ and $[\mathrm{Fe} / \mathrm{H}]_{\mathrm{Kirby}}$. We find that the median offset is

${ }^{3}$ E. Kirby has kindly provided the relevant data so that we could do this analysis.

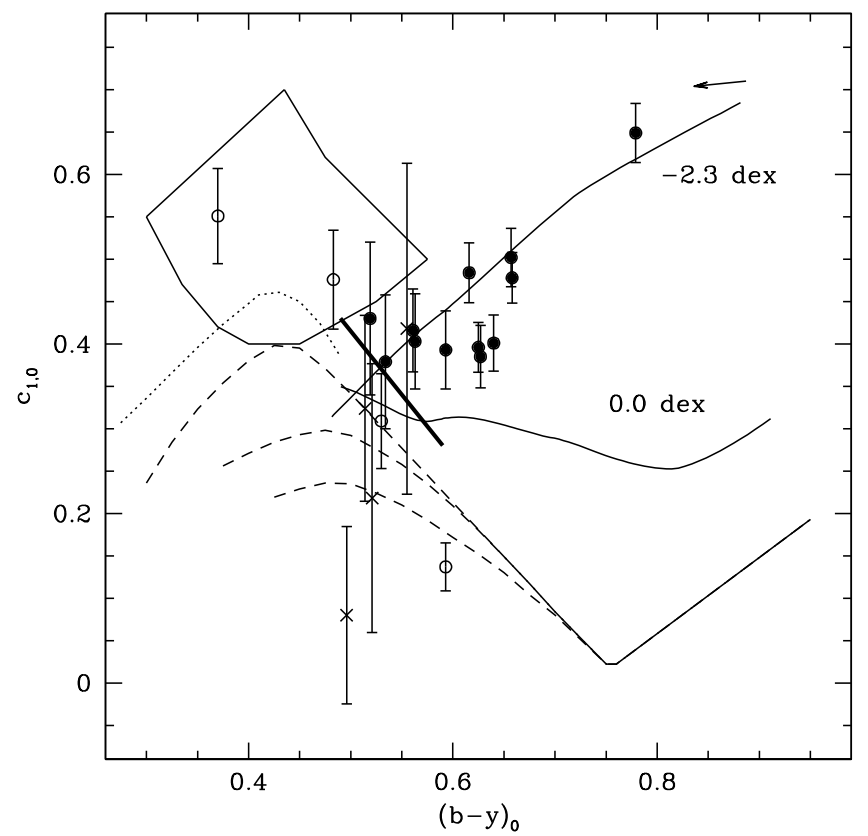

Fig. 24. Strömgren $c_{1,0}$ vs. $(b-y)_{0}$ diagram for the stars from Kirby et al. (2008b). - are stars considered as RGB members and $\circ$ are stars identified as non-RGB members based on our Strömgren photometry (see Sect. 4.2). $\times$ are stars that have $V_{0}>21$, hence we have not considered their evolutionary stage as they are too faint for our membership determination based on Strömgren photometry. The thin solid lines indicates isochrones for RGB stars by VandenBerg et al. (2006) with colour transformations by Clem et al. (2004). The thin dashed lines indicates dwarf star sequences for different metallicities, $[\mathrm{Fe} / \mathrm{H}]=0.45,-0.05$ and -1 top to bottom and the dotted line marks the upper envelope for dwarf stars (all lines from Árnadóttir et al., in preparation). The thick solid line is our lower limit for the identification of RGB stars. The arrow in the top right corner indicates the magnitude and direction of the de-reddening applied to the data (see Sect. 2.3).

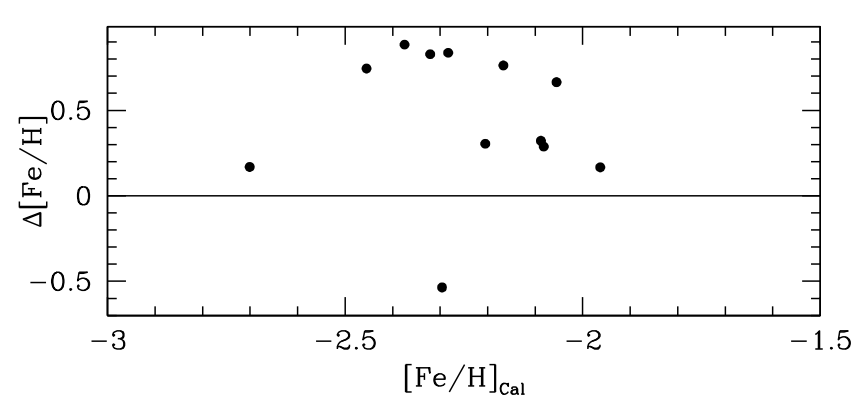

Fig. 25. A comparison between our metallicities derived from $m_{1}$ and metallicities from Kirby et al. (2008b). $\Delta[\mathrm{Fe} / \mathrm{H}]=[\mathrm{Fe} / \mathrm{H}]_{\mathrm{Cal}}-$ $[\mathrm{Fe} / \mathrm{H}]_{\text {Kirby }}$.

$0.67 \mathrm{dex}$, where $[\mathrm{Fe} / \mathrm{H}]_{\mathrm{Cal}}$ is more metal-rich. We have investigated $\Delta[\mathrm{Fe} / \mathrm{H}]$ as a function of both magnitude and radial velocity but found no trend.

Simon \& Geha (2007) obtained metallicities from the Ca II IR triplet lines and found a mean metallicity of $[\mathrm{Fe} / \mathrm{H}]=$ -2.27 dex. Finally, Coleman et al. (2007) report a metallicity of $[\mathrm{Fe} / \mathrm{H}]=-2.26$ dex based on fitting an isochrone to a Colourmagnitude diagram.

In summary, we have five different determinations of the mean metallicity for the Hercules dSph galaxy. These determinations range from about -2.25 dex (Simon \& Geha 2007; 
Table 8. Summary of determination of systemic velocities, velocity dispersions and metallicities for the Hercules dSph galaxy.

\begin{tabular}{llcccc}
\hline \hline & Number of stars & $\begin{array}{c}v_{\text {sys }} \\
{\left[\mathrm{km} \mathrm{s}^{-1}\right]}\end{array}$ & $\begin{array}{c}\sigma \\
{\left[\mathrm{km} \mathrm{s}^{-1}\right]}\end{array}$ & $\begin{array}{c}\langle[\mathrm{Fe} / \mathrm{H}]\rangle \\
\text { dex }\end{array}$ & $\begin{array}{c}{[\mathrm{Fe} / \mathrm{H}]_{\text {Median }}} \\
\text { dex }\end{array}$ \\
\hline This study & 28 [RGB stars, $c_{1}$ sel.] & $\ldots$ & $\ldots$ & $-2.35 \pm 0.31$ & $-2.25_{-0.31}^{+0.14}$ \\
This study & 32 [Only $V_{\text {rad }}$ sel.] & $40.87 \pm 1.42$ & $7.33 \pm 1.08$ & $\ldots$ & $\ldots$ \\
This study & 18 [RGB stars with $\left.V_{\text {rad }}\right]$ & $45.20 \pm 1.09$ & $3.72 \pm 0.91$ & $-2.34 \pm 0.30$ & $-2.25_{-0.20}^{+0.14}$ \\
S\&G & 29 [Their calculation] & $45.0^{a} \pm 1.1$ & $5.1^{a} \pm 0.9$ & $\ldots$ & $\ldots$ \\
S\&G $c_{1}$ & $13\left[c_{1}\right.$ sel. sample] & $46.10 \pm 1.30$ & $4.01 \pm 1.08$ & $\ldots$ & $\ldots$ \\
Kirby et al. & 20 [Their sample] & $\ldots$ & $\ldots$ & $-2.58^{b} \pm 0.51$ & $\ldots$ \\
Kirby et al. $+c_{1}$ & $12\left[c_{1}\right.$ sel. sample] & $\ldots$ & $\ldots$ & $-2.70 \pm 0.47$ & $-2.72_{-0.40}^{+0.35}$ \\
\hline
\end{tabular}

${ }^{a}$ Value from Simon \& Geha (2007). ${ }^{b}$ Value from Kirby et al. (2008b).

Columns 1 and 2 list the number of stars for each study and a short description of how the stars were selected. Note: S\&G indicates the sample of 29 stars from Simon \& Geha (2007). Columns 3 and 4 list the systemic velocities and velocity dispersions and their errors. Column 5 lists the mean metallicities. Column 6 lists the median metallicities. Errors for the median metallicity are the upper and lower quartile. For the calculations we use our metallicites based on Strömgren photometry, but for the Kirby et al. (2008b) sample we use their metallicities.

Coleman et al. 2007, this study) to -2.7 dex (Kirby et al. 2008b). All determinations based on measurements of the $\mathrm{Ca}$ II IR triplet lines and the $m_{1}$-index agree on a mean metallicity of about -2.3 dex. These determinations also agree with the high-resolution analysis by Koch et al. (2008b). The determinations by Kirby et al. (2008b) are $\sim 0.5$ dex more metal-poor (Table 8). This discrepancy is not-negligible. However, future high-resolution studies of additional Hercules stars (as in Koch et al. 2008b, which essentially is in agreement with our method) will determine which methods tend to over- or underestimate the $[\mathrm{Fe} / \mathrm{H}]$. This has important implications for our understanding of the origin of the metal poor halo stars (e.g. Helmi et al. 2006; Koch 2009).

\subsection{Metallicity for the Hercules dSph galaxy}

We find a metallicity range of $-2.99<[\mathrm{Fe} / \mathrm{H}]_{\text {CaII }}<-1.88$ with a mean metallicity of $\left\langle[\mathrm{Fe} / \mathrm{H}]_{\mathrm{Cal}}\right\rangle=-2.35$ dex with $\sigma=$ $0.31 \mathrm{dex}$, and a median $[\mathrm{Fe} / \mathrm{H}]$ of $-2.25 \mathrm{dex}$ with an upper and lower quartile of $-2.11 \mathrm{dex}$ and $-2.56 \mathrm{dex}$, respectively. If we exclude stars fainter than $V_{0}=20.5$ (compare Fig. 22a) we find a mean metallicity of $\left\langle[\mathrm{Fe} / \mathrm{H}]_{\text {Cal }}\right\rangle=-2.26$ dex with $\sigma=0.24$ dex. We do not detect any significant spatial metallicity gradient in our data.

\section{Discussion}

\subsection{Spatial distribution}

The new, faint dSph galaxies are in general found to be quite elongated (Martin et al. 2008). The dSph galaxy in Hercules is no exception, in fact it is one of the new galaxies with the largest ellipticity $(e=0.68)$. The large ellipticity of these objects might be attributable to tidal distortions but could also be due to poor sampling statistics. Each galaxy is only represented by a limited number of RGB stars in most of the cases (Martin et al. 2008). Martin et al. (2008) and Ural \& Wilkinson (2008) conclude that it is entirely possible that the shape determinations for these the faintest of galaxies are entirely dominated by shot-noise. This does not exclude tidal disruption as an explanation for their, in general, very elongated shapes.

With our Strömgren observations we have searched about $2 / 3$ of the area on the sky inside the King profile limiting radius, as defined by Coleman et al. (2007), for members of the Hercules $\mathrm{dSph}$ galaxy. Additionally, we have searched about the same area on the sky outside the King profile limiting radius (see Figs. 1 and 26). Through this search we have found in total 28 stars that are RGB members of the Hercules dSph galaxy. These stars are spatially confined to a fairly small, elongated area falling inside the core radius from Coleman et al. (2007; see Fig. 26a). The RGB members show a slight distortion such that there appear to be a few stars trailing the ellipsoid in the direction of the Milky Way. These stars are few and none have had their radial velocities measured.

The distribution of the HB, RHB, and potential variable stars are shown in Fig. 26b. These stars confirm the central concentration and general shape of the Hercules dSph galaxy. There are some HB, RHB and variable stars scattered outside the ellipsoid defining the King profile limiting radius. It can not be excluded that some of these stars are foreground contaminators. The presence of such stars might distort the determination of the shape parameters for the galaxy. Measurements of radial velocities for these stars would confirm their membership. To our knowledge the HB of the Hercules dSph galaxy has not yet been targeted for such observations.

In conclusion we find that for a well-defined sample of RGB stars (free from contaminating foreground dwarf stars) the Hercules dSph galaxy appears to still have a fairly elongated structure, confirming previous studies.

\subsection{Measured velocity dispersion}

As discussed in Sect. 5, the velocity peak of the Hercules dSph galaxy lies within the bulk of the velocity distribution of the Milky Way galaxy, thus contaminating it. We have shown that relying on radial velocites for membership determination yields a velocity dispersion of $7.33 \pm 1.08 \mathrm{~km} \mathrm{~s}^{-1}$, whilst excluding stars that are not members (i.e. they are foreground dwarf stars) yields a velocity dispersion of $3.72 \pm 0.91 \mathrm{~km} \mathrm{~s}^{-1}$.

It is clear that the velocity dispersion is over-estimated when only considering the radial velocity for membership identification. This is expected since the foreground contaminating stars in the direction of the Hercules dSph galaxy have a much broader velocity distribution than that for the dSph galaxy (compare Fig. 8).

In Fig. 27 we show the velocity distribution for our observations in the velocity range $0<V_{\text {rad }}<100 \mathrm{~km} \mathrm{~s}^{-1}$, highlighting the objects that are members based on the evolutionary stage as derived in Sect. 4.2. As can be seen, objects with a lower velocity than the systemic velocity of the Hercules dSph galaxy are more likely to be excluded as foreground contaminating dwarf 

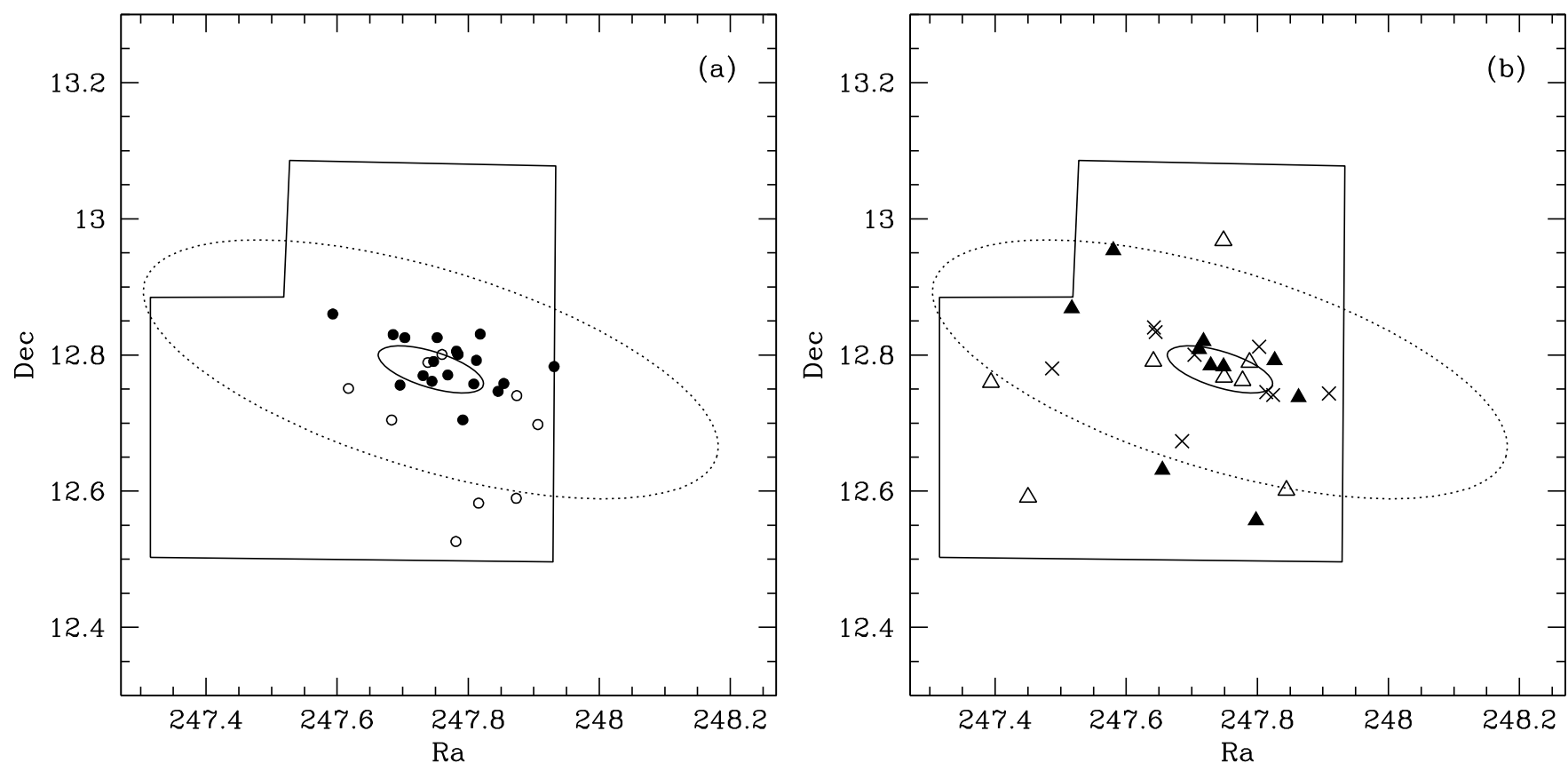

Fig. 26. Spatial distribution of the stars identified as members from both spectroscopy and photometry. a) • are members based on the evolutionary stage as derived from photometry and they have radial velocities within $3 \sigma$ of the systemic velocity for the Hercules dSph galaxy (see Sect. 5.2). The $\circ$ are stars that do not have radial velocity measurements but are members according to our photometric criteria (see Sect. 4.2). b) Filled triangles are HB members. $\triangle$ are probable variable stars and $\times$ are stars identified as RHB-AGB based on the evolutionary stage as derived from photometry (see Sects. 4.5 and 4.3, respectively). The solid ellipse represents the core radius and the dotted ellipse the King profile limiting radius of the Hercules dSph galaxy as determined by Coleman et al. (2007). Solid lines outline the footprint of the WFC. Central coordinates for the galaxy are from Martin et al. (2008).

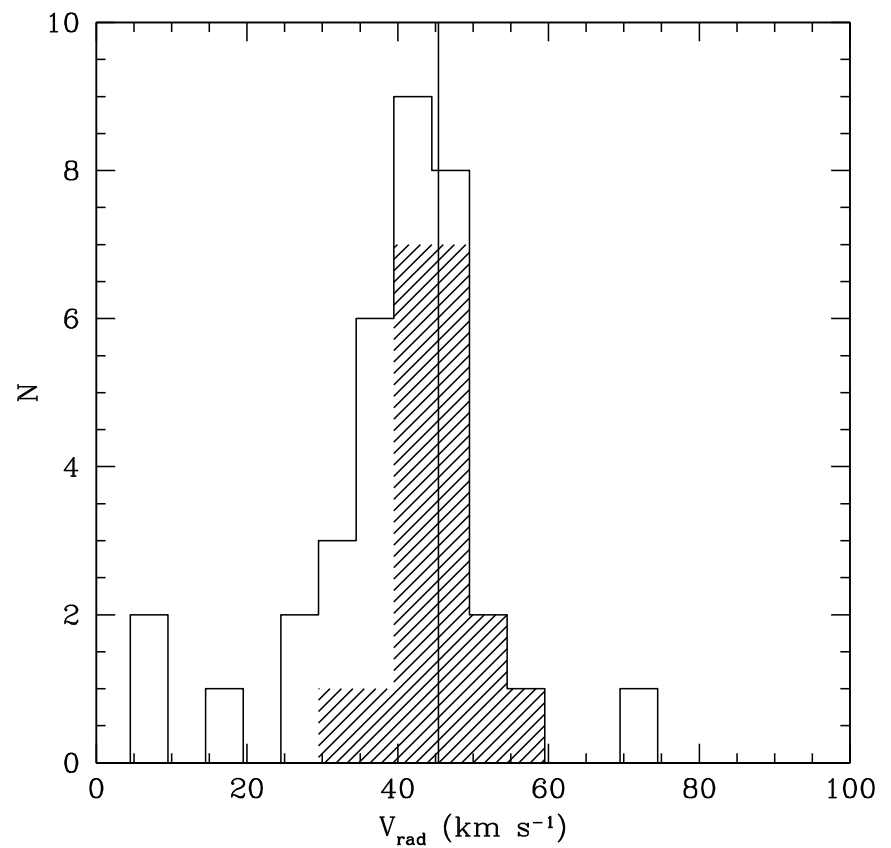

Fig. 27. Distributions of radial velocities. The solid histogram shows the distribution of velocities for objects in the direction of the Hercules dSph galaxy. The shaded histogram shows the distribution of radial velocities for stars identified as RGB members based on the evolutionary stage as derived from photometry. The solid-vertical line indicates the systemic velocity as derived from the RGB members.

stars. Comparing with Fig. 8 verifies that the majority of the velocities from a Besançon model in the direction of the Hercules
$\mathrm{dSph}$ galaxy have a velocity lower than the systemic velocity for dSph galaxy.

In conclusion we find that when deriving the velocity dispersion for $\mathrm{dSph}$ galaxies it is important to have a well defined sample of member stars representing the velocity distribution of the dSph galaxy.

\subsection{Kinematic sub-structure in Hercules.}

Simon \& Geha (2007) detected possible evidence of kinematic sub-structure in the Hercules dSph galaxy. They found nine stars clumped together between 41 and $43 \mathrm{~km} \mathrm{~s}^{-1}$ in a sample of 30 stars distributed between 30 and $60 \mathrm{~km} \mathrm{~s}^{-1}$.

However, for our final sample of RGB stars in the Hercules dSph galaxy a one-sample Kolmogorov-Smirnov test yields a significance level of $74 \%$ for the null hypothesis that the radial velocity distribution is drawn from a Gaussian distribution. The conclusion is therefore that we do not see any kinematic substructure in our sample.

\subsection{Metallicity}

Figure 28a shows the metallicity distribution for the Hercules dSph galaxy based on the $m_{1}$-index for our final sample of RGB members (see Sects. 6 and 8.1). Figure 28b shows the corresponding normal probability function. The data points only deviate slightly from a linear fit which suggests that the metallicity distribution is drawn from a single normal distribution. The slight deviation from the linear fit is noticeable at low metallicities where the cut-off is less sharp than at high metallicities. These features is predicted by models and indicate the occurrence of strong galactic winds (Lanfranchi \& Matteucci 2007). 


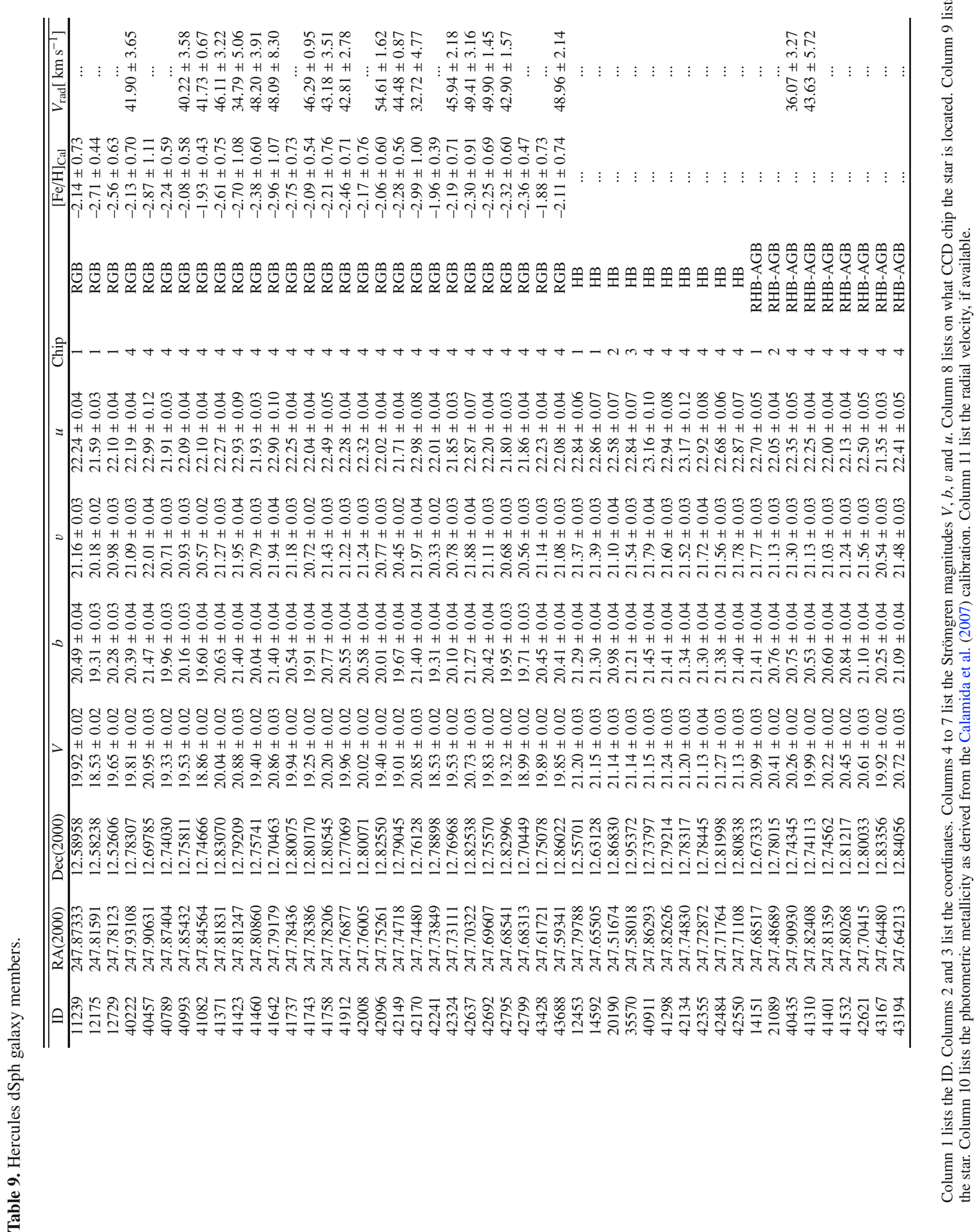




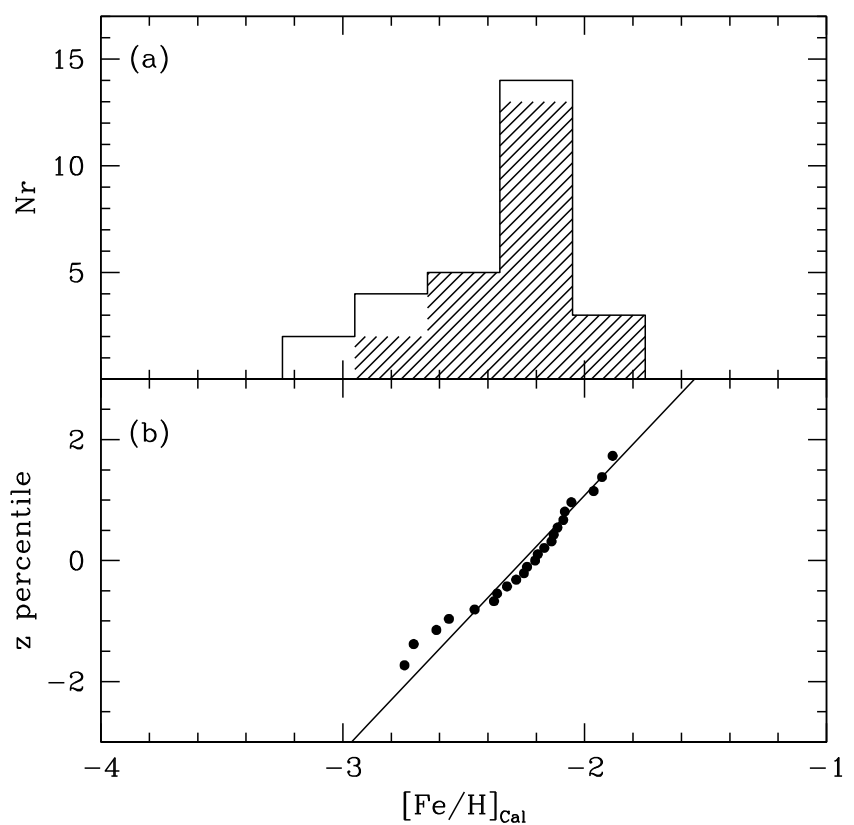

Fig. 28. a) Metallicity histogram for RGB stars in the Hercules dSph galaxy. The solid histogram shows the distribution for all stars in our final sample. The shaded histogram shows the distribution for stars in our final sample with $V_{0}<20.5$ (compare Fig. 22a). b) Corresponding normal probability plot, for stars in our final sample with $V_{0}<20.5$, assuming a normal distribution. The solid line indicates a linear fit to the data with a mean metallicity of $[\mathrm{Fe} / \mathrm{H}]=-2.26$ dex and $\sigma=0.24$ dex.

In Sect. 8.1.2 we calculated the errors in $[\mathrm{Fe} / \mathrm{H}]_{\mathrm{Cal}}$. When we take into account the uncertainties in zeropoints, extinction coefficients and colour terms we note that the errors are in general much larger than the $\sigma$ of the metallicity distribution (compare Fig. 22a where we show the error in $\left.[\mathrm{Fe} / \mathrm{H}]_{\mathrm{Cal}}\right)$. However, since the uncertainties in zeropoints, extinction coefficients and colour terms propagate as a magnitude offset, equal for all stars, we re-calculate the errors in metallicities excluding the uncertainties in zeropoints, extinction coefficients and colour terms (see Fig. 22a). We find that these errors in metallicity, for stars brighter than $V_{0}=20.5$, are smaller than the metallicity spread of 0.24 dex. This enables us to study the star-to-star scatter in $[\mathrm{Fe} / \mathrm{H}]_{\mathrm{Cal}}$, making the profile of the distribution in Fig. 28 significant. We conclude that there is an abundance spread in the metallicity distribution for the RGB members of up to 1 dex.

\section{Summary}

We have, for the first time, presented a list of Hercules dSph galaxy members based on an analysis of radial velocity, evolutionary stage obtained from photometry, and stellar classification using SExtractor. In detail we provide the following inventory of the Hercules dSph galaxy

- 28 stars as RGB members based on their evolutionary stage, see Sect. 4.2. Of these, 19 have measured radial velocities (see Sect. 5.2).

- 9 stars as RHB-AGB members based on their evolutionary stage (see Sect. 4.2). Of these, 2 have radial velocities (see Sect. 5.2).

- 10 stars as BHB members based on their evolutionary stage, see Sect. 4.2.

- 8 stars that are possible variable stars based on their evolutionary stage, see Sect. 4.5.
Our best determination of the systemic velocity is $45.20 \pm$ $1.09 \mathrm{~km} \mathrm{~s}^{-1}$ with a dispersion of $3.72 \pm 0.91 \mathrm{~km} \mathrm{~s}^{-1}$. We have shown that membership based on radial velocity alone is not a good method for the Hercules dSph galaxy, since it has a systemic velocity that falls well within the velocity distribution of the foreground dwarf stars belonging to the Milky Way.

Stellar metallicities have been determined using the Strömgren $m_{1}$ index with a calibration that translates $m_{1,0}$ to $[\mathrm{Fe} / \mathrm{H}]$ for $\mathrm{RGB}$ stars. We found a mean metallicity of -2.35 dex. We also derived metallicities for our stars observed with FLAMES from measurements of the equivalent width of the Ca II IR triplet lines. The agreement between the two determinations was very good, with an offset of only 0.06 dex.

Finally, we have estimated the mean magnitude of the HB of the Hercules dSph galaxy to $V_{0}=21.17 \pm 0.05$ based on the 10 stars identified as BHB members. This gives a distance of $147_{-7}^{+8} \mathrm{kpc}$.

Acknowledgements. We would like to thank Andreas Korn for putting us on the right track to understand the erroneous spectra created by the CCD-glow. D.A. thanks Simon Hodgkin at the Wide Field Survey unit at IoA, Cambridge, for help with the INT data reduction. D.A. thanks Lennart Lindegren at Lund Observatory for his help in problems of mathematical nature. S.F. is a Royal Swedish Academy of Sciences Research Fellow supported by a grant from the Knut and Alice Wallenberg Foundation. M.I.W. is supported by a Royal Society University Research Fellowship.

Funding for the SDSS and SDSS-II has been provided by the Alfred P. Sloan Foundation, the Participating Institutions, the National Science Foundation, the US Department of Energy, the National Aeronautics and Space Administration, the Japanese Monbukagakusho, the Max Planck Society, and the Higher Education Funding Council for England. The SDSS Web Site is http://www . sdss.org/. The SDSS is managed by the Astrophysical Research Consortium for the Participating Institutions. The Participating Institutions are the American Museum of Natural History, Astrophysical Institute Potsdam, University of Basel, University of Cambridge, Case Western Reserve University, University of Chicago, Drexel University, Fermilab, the Institute for Advanced Study, the Japan Participation Group, Johns Hopkins University, the Joint Institute for Nuclear Astrophysics, the Kavli Institute for Particle Astrophysics and Cosmology, the Korean Scientist Group, the Chinese Academy of Sciences (LAMOST), Los Alamos National Laboratory, the Max-Planck-Institute for Astronomy (MPIA), the Max-Planck-Institute for Astrophysics (MPA), New Mexico State University, Ohio State University, University of Pittsburgh, University of Portsmouth, Princeton University, the United States Naval Observatory, and the University of Washington.

\section{References}

Anthony-Twarog, B. J., \& Twarog, B. A. 1994, AJ, 107, 1577

Battaglia, G., Irwin, M., Tolstoy, E., et al. 2008, MNRAS, 383, 183

Belokurov, V., Zucker, D. B., Evans, N. W., et al. 2006, ApJ, 647, L111

Belokurov, V., Zucker, D. B., Evans, N. W., et al. 2007, ApJ, 654, 897

Belokurov, V., Walker, M. G., Evans, N. W., et al. 2008, ApJ, 686, L83

Belokurov, V., Walker, M. G., Evans, N. W., et al. 2009, MNRAS, 397, 1748

Bertin, E., \& Arnouts, S. 1996, A\&AS, 117, 393

Blecha, A., Cayatte, V., North, P., Royer, F., \& Simond, G. 2000, in Optical and IR Telescope Instrumentation and Detectors, ed. M. Iye, \& A. F. Moorwood, Proc. SPIE, 4008, 467

Bonanos, A. Z., Stanek, K. Z., Szentgyorgyi, A. H., Sasselov, D. D., \& Bakos, G. Á. 2004, AJ, 127, 861

Bond, H. E. 1980, ApJS, 44, 517

Calamida, A., Bono, G., Stetson, P. B., et al. 2007, ApJ, 670, 400

Carretta, E., \& Gratton, R. G. 1997, A\&AS, 121, 95

Carretta, E., Gratton, R. G., Clementini, G., \& Fusi Pecci, F. 2000, ApJ, 533, 215

Clem, J. L., VandenBerg, D. A., Grundahl, F., \& Bell, R. A. 2004, AJ, 127, 1227

Cole, A. A., Smecker-Hane, T. A., Tolstoy, E., Bosler, T. L., \& Gallagher, J. S. 2004, MNRAS, 347, 367

Coleman, M. G., de Jong, J. T. A., Martin, N. F., et al. 2007, ApJ, 668, L43 Faria, D., Feltzing, S., Lundström, I., et al. 2007, A\&A, 465, 357

Gilbert, K. M., Guhathakurta, P., Kalirai, J. S., et al. 2006, ApJ, 652, 1188

Gilmore, G., Wilkinson, M. I., Wyse, R. F. G., et al. 2007, ApJ, 663, 948

Grebel, E. K., \& Richtler, T. 1992, A\&A, 253, 359

Grebel, E. K., Gallagher, III, J. S., \& Harbeck, D. 2003, AJ, 125, 1926

Helmi, A., Irwin, M. J., Tolstoy, E., et al. 2006, ApJ, 651, L121 
Hilker, M. 2000, A\&A, 355, 994

Howell, S. B. 1989, PASP, 101, 616

Irwin, M., \& Lewis, J. 2001, New Astron. Rev., 45, 105

Jordi, K., Grebel, E. K., \& Ammon, K. 2006, A\&A, 460, 339

Keller, S. C., Schmidt, B. P., Bessell, M. S., et al. 2007, Publications of the Astronomical Society of Australia, 24, 1

Kirby, E. N., Guhathakurta, P., \& Sneden, C. 2008a, ApJ, 682, 1217

Kirby, E. N., Simon, J. D., Geha, M., Guhathakurta, P., \& Frebel, A. 2008b, ApJ, $685, \mathrm{~L} 43$

Kleyna, J., Wilkinson, M. I., Evans, N. W., Gilmore, G., \& Frayn, C. 2002, MNRAS, 330, 792

Kniazev, A. Y., Grebel, E. K., Pustilnik, S. A., Pramskij, A. G., \& Zucker, D. B. 2005, AJ, 130, 1558

Koch, A. 2009, ArXiv e-prints

Koch, A., Grebel, E. K., Wyse, R. F. G., et al. 2006, AJ, 131, 895

Koch, A., Grebel, E. K., Kleyna, J. T., et al. 2007a, AJ, 133, 270

Koch, A., Wilkinson, M. I., Kleyna, J. T., et al. 2007b, ApJ, 657, 241

Koch, A., Grebel, E. K., Gilmore, G. F., et al. 2008a, AJ, 135, 1580

Koch, A., McWilliam, A., Grebel, E. K., Zucker, D. B., \& Belokurov, V. 2008b, ApJ, 688, L13

Koch, A., Rich, R. M., Reitzel, D. B., et al. 2008c, ApJ, 689, 958

Lanfranchi, G. A., \& Matteucci, F. 2007, A\&A, 468, 927

Lind, K., Korn, A. J., Barklem, P. S., \& Grundahl, F. 2008, A\&A, 490, 777

Marigo, P., Girardi, L., Bressan, A., et al. 2008, A\&A, 482, 883

Martin, N. F., de Jong, J. T. A., \& Rix, H.-W. 2008, ApJ, 684, 1075

Olsen, E. H. 1983, A\&AS, 54, 55

Olsen, E. H. 1984, A\&AS, 57, 443

Olsen, E. H. 1993, A\&AS, 102, 89
Olsen, E. H. 1994a, A\&AS, 104, 429

Olsen, E. H. 1994b, A\&AS, 106, 257

Olsen, E. H. 1995, A\&A, 295, 710

Olszewski, E. W., Pryor, C., \& Armandroff, T. E. 1996, AJ, 111, 750

Pasquini, L., Avila, G., Blecha, A., et al. 2002, The Messenger, 110, 1 Richtler, T. 1989, A\&A, 211, 199

Robin, A. C., Reylé, C., Derrière, S., \& Picaud, S. 2003, A\&A, 409, 523

Rutledge, G. A., Hesser, J. E., \& Stetson, P. B. 1997a, PASP, 109, 907

Rutledge, G. A., Hesser, J. E., Stetson, P. B., et al. 1997b, PASP, 109, 883

Schiavon, R. P., Barbuy, B., Rossi, S. C. F., \& Milone, A. 1997, ApJ, 479, 902

Schlegel, D. J., Finkbeiner, D. P., \& Davis, M. 1998, ApJ, 500, 525

Schuster, W. J., \& Nissen, P. E. 1988, A\&AS, 73, 225

Schuster, W. J., Beers, T. C., Michel, R., Nissen, P. E., \& García, G. 2004, A\&A, 422,527

Shetrone, M. D., Côté, P., \& Sargent, W. L. W. 2001, ApJ, 548, 592

Siegel, M. H. 2006, ApJ, 649, L83

Simon, J. D., \& Geha, M. 2007, ApJ, 670, 313

Strigari, L. E., Bullock, J. S., Kaplinghat, M., et al. 2008, Nature, 454, 1096 Tonry, J., \& Davis, M. 1979, AJ, 84, 1511

Ural, U., \& Wilkinson, M. 2008, Astron. Nachr., 329, 1040

VandenBerg, D. A., Bergbusch, P. A., \& Dowler, P. D. 2006, ApJS, 162, 375

Walker, M. G., Mateo, M., Olszewski, E. W., et al. 2006, AJ, 131, 2114

Walker, M. G., Mateo, M., Olszewski, E. W., et al. 2007, ApJ, 667, L53

Walsh, S. M., Jerjen, H., \& Willman, B. 2007, ApJ, 662, L83

Walsh, S. M., Willman, B., \& Jerjen, H. 2009, AJ, 137, 450

Zinn, R., \& West, M. J. 1984, ApJS, 55, 45

Zucker, D. B., Belokurov, V., Evans, N. W., et al. 2006a, ApJ, 650, L41

Zucker, D. B., Belokurov, V., Evans, N. W., et al. 2006b, ApJ, 643, L103 